\title{
Research Policy and Endogenous Growth
}

\author{
María Fuensanta Morales* \\ Universitat Autònoma de Barcelona y Universidad de Murcia. \\ Departamento de Fundamentos del Análisis Económico. \\ Facultad de Economía y Empresa. \\ Campus de Espinardo. \\ Universidad de Murcia. \\ Espinardo. 30100 Murcia Spain. \\ Phone: +34 968367 910. Fax: +34968 363758 \\ e-mail: fmorales@um.es
}

Keywords: Research policy; basic and applied research; endogenous growth.

JEL codes: O31; O38; O40.

April, 5th 2001

\begin{abstract}
This paper studies the effects of different types of research policy on economic growth. We find that while tax incentives to private research, public funding of private projects, and basic research performed at public institutions have unambiguously positive effects on economic growth, performing applied research at public institutions could have negative growth effects. This is due to the large crowding out of private research caused by public $R \& D$ when it competes with private firms in the "patent race". Concerning the effects of these policies on welfare, it is found that research policy can either improve or reduce consumer welfare depending on the characteristics of the policy and that an excessively high research subsidy will reduce it.
\end{abstract}

${ }^{*}$ I am thankful to Jordi Caballé for his valuable comments and directions. I would also like to aknowledge the help of David Pérez-Castrillo with the initial design of the $R \& D$ sector. 


\section{Introduction}

The objective of this paper is to study the effect of public research policy on both the productivity of private $R \& D$ and the growth performance of the economy. In order to do so, we will consider different research policies in the context of an endogenous growth model, where we make explicit the difference between basic and applied research.

Previous literature on public intervention in the research sector is mainly undertaken from the industrial organization perspective. Papers on this area are generally concerned with the microeconomic effects of research subsidies and patent policy. Some attention has been paid, however, to public research. The papers by Mamuneas and Nadiri (1996), Ham and Mowery (1998) and Mamuneas (1999) provide microeconomic foundations for the hypothesis that public R\&D causes positive external effects on private productivity. In addition, Mamuneas and Nadiri (1996) find econometric evidence that publicly financed R\&D induces cost savings but crowds out privately financed $R \& D$ investment.

There are few papers that consider public research investment from a macroeconomic perspective. Glomm and Ravikumar (1994) present a model in which the economy grows thanks to public research. However, this paper is focussed on distributional problems and, therefore, the presence of public research in this model is just a simplifying assumption in order to obtain endogenous technological innovation without the difficulties that would imply the introduction of a private R\&D sector. Pelloni (1997) allows the government to invest in public research so as to improve the growth performance of the economy but does not allow for private research. On the contrary, Park (1998) considers both public and private research. This author introduces public research in the model of expanding variety of products first presented in Romer (1990). He assumes that public research indirectly contributes to economic growth because it causes a positive external effect on the knowledge accumulation of the private sector. However, the paper is mainly concerned with open economies issues and international spillovers rather than with public research policy. This last paper does not distinguish between basic and applied research. Indeed, the difference between basic and applied research is absent from all the papers previously mentioned. Very few authors have tried to address the issue of basic versus applied research, especially in a macroeconomic context. The paper by David (2000) reviews the literature and establishes the main debates on the issue of public science, focusing on the differences between basic and applied research and the need for public provision of basic knowledge. Similarly, the work of David and Hall (2000) analyzes the effects of the various public research policies on private R\&D expenditures, though the analysis is performed by means of a simple, partial equilibrium, static model. Regarding empirical studies on the influence of R\&D expenditures on productivity growth, Griliches (1986) finds evidence of the positive effects of both publicly financed R\&D and basic research while Mansfield (1995) analyses the interaction between academic research and industrial innovation. The most recent econometric work on the relationship between public and private research is surveyed in David, Hall and Toole (2000). However, there still exists a need for a theoretical model able to modelize the effects of research policy on economic growth.

In order to bring the analysis closer to reality, we have considered the main policy responses that actual 
governments use to prevent private underprovision of research. These policies are usually classified in two groups. The first one concerns the direct procurement of research in public facilities, while the second includes policies consisting on giving incentives for a greater amount of private investment. These incentives can take the form of tax reductions intended to reduce the cost of R\&D but they can also involve direct funding of specific R\&D programs. We will modelize these types of policies in the framework of an endogenous growth model.

There exists a growing debate concerning whether public research should take the form of basic or applied research and whether it should be performed at public institutions or in close coordination with private firms. We will make explicit the difference between basic and applied research and explore the different effects that the various policies available could have on the $\mathrm{R} \& \mathrm{D}$ sector and the economy as a whole.

The basic model we propose as framework for this analysis is the one first presented in Aghion and Howitt (1998). In this model, the economy grows thanks to both capital accumulation and technological change. Therefore, this model overcomes one of the main objections traditionally raised against technological change models, namely, that capital accumulation was ignored as a source of growth. Furthermore, the presence of a continuum of research sectors eliminates uncertainty at the aggregate, allowing for the use of non-stochastic steady state analysis at the macroeconomic level. In Aghion and Howitt model, firms invest in research projects that yield a new product or a new production technology with a certain probability. In the present model, we want to introduce a distinction between basic and applied research. Intuitively, applied research is aimed at obtaining innovations able to improve a particular production technology or that can give raise to a new product or variety. On the contrary, basic research is usually concerned with projects whose outcomes do not normally have a direct market application, though they add to the knowledge base. This does not necessarily mean that private firms will not perform basic research, since we consider that even though basic research alone would not be able to produce a marketable innovation, it is able to increase the productivity of applied research. This is due to the fact that basic science allows researchers in applied fields to understand previous knowledge or to adapt innovations from other fields to their own sector.

We find that subsidies to private research increase R\&D investment, both in applied and basic fields, and that this policy is beneficial for long term growth. However, the effect on steady state consumption is generally negative and, therefore, the final effect on welfare results from the trade off between consumption and growth. Due to this fact, the sign of the effect on welfare is ambiguous. For a empirically acceptable set of parameter values, a marginal subsidy to research would have positive effects though excessively high values of the subsidy could harm welfare rather than improve it.

Concerning the other available policy instruments, we will differentiate public production of research from direct funding of $R \& D$ projects in the following manner: when research is performed at public institutions, any innovation with a market application that arises from public research will compete with private research in the concession of patents. On the other hand, direct funding of research consists of public aids to private projects which, if successful, will keep the patent in the private sector. Consider thus first the case in which the public sector performs exclusively basic research at public institutions. This type of research increases 
aggregate knowledge and will affect private firms only through the spillovers created by the faster growing base of knowledge. In other words, the faster accumulation of non-rival and non-excludable knowledge will induce a more important technology improvement when an innovation occurs in the private sector. The growth effect of a higher public budget for research is unambiguously positive while the welfare effect, calibrated for empirically acceptable values of the parameters, seems to be also positive.

When the public sector is allowed to perform both applied and basic research, it may happen that a public project gains the patent in a given sector. In this sense, the public sector behaves as a direct competitor of private research firms and therefore, the public investment in research should be taken into account when computing the sector's rate of replacement. This rate is given by the probability that an innovation occurs in a given sector, which in turn is determined by the amount of research invested in that sector. A higher rate of replacement implies a lower value of the innovation because it reduces the expected life of the patent. Therefore, even though public research will add to the accumulation of knowledge, it also causes this "business-stealing" effect that crowds out private research. Consequently, the net result on the growth rate will depend on which effect dominates and on the actual values of private and public research. We find that in order to have a positive effect on growth of either type of public R\&D, the amounts of public applied and basic research must keep certain proportions. In particular, we find that increasing public applied research from zero, the crowding out of private research is so large that the effect on growth will initially be negative for any given value of public basic research. However, if we keep increasing public applied research, the effect on growth will become positive. This is due to the fact that public research is actually substituting private $R \& D$ as the source of innovative activity. Indeed, it is relatively easy to crowd out completely private research when the public sector performs both applied and basic research. With regard to the welfare change induced by this policy, again the opposite behavior of consumption and growth forces a calibration in order to obtain a sign. The calibration suggests that welfare may be improved with both types of public research though the introduction of applied public research will initially reduce welfare. Similarly, excessive amounts of public research will determine low levels of steady state consumption per efficiency unit which eventually, will impede further welfare improvements.

An alternative policy instrument is direct funding of specific research projects. In order to simplify and to differentiate it clearly from the previous policy, we assume that the government provides a given amount of output to be used in a specific project, either applied or basic, but that in case of success, the patent remains with the research firm. The implications of this type of financing differ from the previous policy in the sense that the amount of research financed by the public sector increases the productivity of private research. This is so because in order to obtain a given probability of success, the private investment required is smaller the larger the amount financed by the government. We still find the "business stealing" effect of the previous policy but it is now softened by the increase of research productivity. Consequently, the effects on growth of both applied and basic research are unambiguously positive. Remarkably, we find that in equilibrium, the effects on growth and research intensity of public basic and applied research are identical, which suggests that if the research policy is developed through direct funding of private projects, the relevant 
amount is the total research investment and not whether it has been devoted to applied or basic projects. Another relevant difference with respect to public production of research is the impact on the amount of private investment in research. While research performed at public institutions causes a clear crowding out of private R\&D investment, the effect of direct funding is ambiguous. Depending on the actual values of the parameters, we can even find that public and private research behave as complements at the steady state. The econometric evidence is not clear at this point. While some studies identify public and private research as substitutes, other works find that an increase in public research may cause a parallel increase in private $R \& D$ investment. ${ }^{1}$ The fact that data on $R \& D$ expenditures usually include together both public research and public funding of private projects may be one of the causes of the present difficulties to settle the question. With respect to the welfare effects of this policy, again the trade off between consumption and growth determines a positive impact on welfare for small values of public research investment that may become negative for higher values as consumption per efficiency unit diminishes.

In summary, the results suggest that while tax incentives to private research always have a positive growth effect, public research may not be the appropriate policy in some circumstances. We find that research performed at public institutions is always beneficial if it is only concerned with basic research. However, if public institutions do investigate also in applied fields, the impact on long run growth may be negative when some conditions are met. The condition for a positive growth effect requires that the relative amounts of applied and basic research lie between some limits, and that one of them is not excessive with respect to the other. For instance, if the amounts of basic and applied research are chosen so as to maximize the probability of success for a given amount of total investment, the effect on growth is always positive, though the crowding out of private research is so important that it would be relatively easy to crowd it out completely. We also find that direct funding of research has unambiguously positive growth effects.

The rest of the paper is divided into the following sections: section 2 presents the model, sections 3 and 4 present the steady state and welfare analysis and section 5 concludes the paper.

\section{The model}

We consider a growth model with endogenous technological change in which research may be performed by both the private and the public sector. Long run economic growth comes from both technological innovation and capital accumulation. There exist two types of research projects depending on whether they are concerned with basic or applied issues. Successful applied projects produce a new technology that will generate monopoly rents for the owner of the patent. Research projects focused exclusively on basic fields are not able to generate a new product or variety though they contribute to the accumulation of general knowledge. In combination with applied research, basic research is able to increase R\&D productivity because it facilitates the absorption of intersectoral and intertemporal spillovers. As a consequence, private firms will only engage in projects with an applied component though they may find it optimal to devote

\footnotetext{
${ }^{1}$ See David et al (2000).
} 
some additional resources to basic research in order to increase the productivity of their own research.

\subsection{Consumers}

There exists an infinitely lived representative consumer whose utility function is assumed to be logarithmic for the sake of simplicity. Consequently, the lifetime utility of the consumer will be given by the following expression:

$$
V\left(C_{t}\right)=\int_{0}^{\infty} \ln \left(C_{t}\right) e^{-\rho t} d t
$$

where $C_{t}$ is consumption at time $t$ and $\rho$ is the rate of discount.

\subsection{Final good sector}

The consumption good is produced in a competitive market out of labor and intermediate goods. Labor is represented by a continuous mass of individuals $L$, and it is assumed to be inelastically supplied. Intermediate goods are produced by a continuum of sectors of mass 1 , being $m_{i t}$ the supply of sector $i$ at date $t$. The production function is a Cobb-Douglas with constant returns on intermediate goods and efficiency units of labor

$$
Y_{t}=L^{1-\alpha} \int_{0}^{1} A_{i t} m_{i t}^{\alpha} d i
$$

where $Y_{t}$ is final good production and $A_{i t}$ is the productivity coefficient of each sector. The evolution of each sector's productivity coefficient $A_{i t}$ is determined in the research sector. I assume equal factor intensity to simplify calculations.

\subsection{Intermediate goods}

Intermediate goods are used as factors of production in the final good sector. Each sector has a monopolistic structure. In order to become the monopolist producer of an intermediate good, the entrepreneur has to buy the patent of the latest version of the product. This patent gives him the right to produce the good until an innovation occurs and the monopolist is displaced by the owner of the new technology.

The only input in the production of intermediate goods is capital. In particular, it is assumed that $A_{i t}$ units of capital are needed to produce one unit of intermediate good $i$ at date $t$. This implies that more productive intermediate inputs are more capital intensive, an assumption that simplifies the analysis and has no important implications under the Cobb-Douglas conditions.

Capital is rented in a perfectly competitive market at rate $\zeta_{t}$. Hence, the cost of one unit of intermediate good is $A_{i t} \zeta_{t}$. On the other hand, the equilibrium price of the intermediate good, $p\left(m_{i t}\right)$ will be its marginal product

$$
p\left(m_{i t}\right)=\alpha L^{1-\alpha} A_{i t} m_{i t}^{\alpha-1}
$$


where $m_{i t}$ is production of intermediate good $i$ at date $t$. Thus, the monopolist's profit maximization problem is the following:

$$
\begin{aligned}
\pi_{i t} & =\max _{m_{i t}}\left[p\left(m_{i t}\right) m_{i t}-A_{i t} \zeta_{t} m_{i t}\right] \\
\text { subject to } p\left(m_{i t}\right) & =\alpha L^{1-\alpha} A_{i t} m_{i t}^{\alpha-1},
\end{aligned}
$$

from where we obtain the profit-maximizing supply and the flow of profits as

$$
\begin{aligned}
m_{i t} & =L\left(\frac{\alpha^{2}}{\zeta_{t}}\right)^{\frac{1}{1-\alpha}} \\
\pi_{i t} & =\alpha(1-\alpha) L^{1-\alpha} A_{i t} m_{i t}^{\alpha} .
\end{aligned}
$$

Due to the assumption of equal factor intensity, supply of intermediate goods is equal in all sectors, $m_{i t}=m_{t}$. Thus, the aggregate demand of capital is equal to $\int_{0}^{1} A_{i t} m_{t} d i$. Let $A_{t}=\int_{0}^{1} A_{i t} d i$, be the aggregate productivity coefficient. Then, equilibrium in the capital market requires demand to equal supply

$$
A_{t} m_{t}=K_{t}
$$

or equivalently, the flow of intermediate output must be equal to capital intensity, $k_{t}$

$$
m_{t}=k_{t}=\frac{K_{t}}{A_{t}}
$$

With this notation we can express the equilibrium rental rate in terms of capital intensity

$$
\zeta_{t}=\alpha^{2} L^{1-\alpha} k_{t}{ }^{\alpha-1}
$$

\subsection{Research sector}

For each of the above intermediate sectors, there is a number of research firms competing in a patent race to get the next innovation. Innovations are produced using the same technology of the final good. Hence, it needs physical capital (embodied in the intermediate goods) apart from labor to be produced. Technology is assumed to be increasingly complex and hence further innovations will require higher investments. Accordingly, the amount invested in research in each sector $N_{i t}$ will be adjusted by a coefficient representing the aggregate state of knowledge. This coefficient will be given by $A_{t}^{\max }$, the productivity parameter of the leading edge technology. Hence, we may define $n_{i t}=\frac{N_{i t}}{A_{t}^{\text {max }}}$ as the productivity adjusted level of research.

The arrival rate of innovations at each sector is given by the following expression:

$$
\left[\lambda n_{a}\left(1+b n_{b}\right)\right]^{\frac{1}{2}},
$$

where $\lambda$ is a positive parameter representing the productivity of research, $n_{a}$ and $n_{b}$ are the levels of research intensity devoted to applied and basic issues respectively, and $b$ is a positive parameter that measures the influence of basic research on the total private research productivity. ${ }^{2}$ This functional form tries to capture

\footnotetext{
${ }^{2}$ For the functional form of the contributions of basic and applied research to the probability of success we follow Cassiman, Pérez-Castrillo and Veugelers (2001).
} 
the idea that basic research is not essential in order to obtain an innovation with market applications as opposed to applied research, which is assumed to be essential. Given the total amount $n_{i t}$ of research, the firm will choose $n_{a}$ and $n_{b}$ in order to maximize the probability of obtaining an innovation. The optimal shares are thus,

$$
\begin{aligned}
& n_{a}= \begin{cases}\frac{n_{i t}}{2}+\frac{1}{2 b} & \text { if } n_{i t}>\frac{1}{b} \\
n_{i t} & \text { otherwise. }\end{cases} \\
& n_{b}= \begin{cases}\frac{n_{i t}}{2}-\frac{1}{2 b} & \text { if } n_{i t}>\frac{1}{b} \\
0 & \text { otherwise. }\end{cases}
\end{aligned}
$$

In order to simplify the analysis, we will consider only those situations in which private firms invest in basic research, that is, $n_{i t}>\frac{1}{b}{ }^{3}$ The results when this assumption is not satisfied are presented in Appendix D.

In equilibrium, the arrival rate of innovations in sector $i$ will be given by the following expression

$$
\lambda p\left(n_{i t}\right)=\lambda\left(\frac{1+b n_{i t}}{2 \sqrt{b}}\right),
$$

which may be obtained substituting (5) and (6) in (4).

The payoff to innovators if they succeed is the flow of profits obtained from the monopolistic exploitation of the new technology. The value of this payoff is identical for any researcher innovating at $t$ and therefore, research intensity will be the same across sectors. Consequently, we drop the $i$ subindex from research intensity.

When an innovation occurs in a given sector, the productivity parameter of that sector jumps discontinuously to $A_{t}^{\max }$, the leading edge productivity coefficient. Thus, advances in other sectors spillover to the rest of the economy making the technology improvement induced by the next innovation more important. The evolution of $A_{t}^{\max }$ is determined by the evolution of the aggregate state of knowledge. While for a particular firm we assumed that basic research was not essential in order to obtain an innovation, in the case of the aggregate state of knowledge, we are going to assume that both basic and applied research are essential factors. This assumption reflects the extended belief that in the long run, the knowledge base cannot go on growing if basic knowledge is not further developed. ${ }^{4}$ Consequently, we assume that the rate of growth of $A_{t}^{\max }$ is given by the following expression:

$$
\frac{\dot{A}_{t}^{\max }}{A_{t}^{\max }}=\sigma \lambda\left(n_{A}\right)^{\beta}\left(n_{B}\right)^{1-\beta}
$$

where $n_{A}$ and $n_{B}$ are total applied and basic research intensity, that is, including both public and private research. Under these assumptions, the distribution of productivity parameters across sectors will change as $A_{t}^{\max }$ grows. However, if we define the relative productivity parameter of a sector as $a_{i t}=\frac{A_{i t}}{A_{t}^{\text {max }}}$, one can prove that the distribution of $a_{i t}$ converges to a stationary distribution. In addition, the stationarity of the distribution of $a$ implies that the aggregate and the leading edge productivities are proportional. ${ }^{5}$

\footnotetext{
${ }^{3}$ See Appendix D for the parameter restrictions necessary to guarantee this condition.

${ }^{4}$ See David (2000).

${ }^{5}$ For the distribution of relative productivities across sectors see Appendix A.

Let $h(a)$ be the density function of $a$. Then, by definition, $A_{t}=\int_{0}^{1} A_{i t} d i=A_{t}^{\max } \int_{0}^{1} a_{i t} d i=A_{t}^{\max } \int_{0}^{1} a h(a) d a=A_{t}^{\max } E(a)$.
} 
In order to determine private research intensity, consider the value of obtaining an innovation at time $t$. When the innovation occurs, a new technology with a productivity parameter $A_{t}^{\max }$ is available for the owner of the patent. The new producer will force the previous incumbent out of the market and will start producing as a monopolist. Therefore, the flow of profits will be given by the following expression:

$$
\alpha(1-\alpha) L^{1-\alpha} A_{t}^{\max } k_{t}^{\alpha} .
$$

The new producer will be able to keep its monopolistic position until a new innovation occurs in that sector. Therefore, the present value of the innovation at time $t$ is given by

$$
V_{t}=\int_{t}^{\infty} e^{-\int_{t}^{\tau}\left[r_{s}+\lambda p\left(n_{i t}\right)\right] d s}(1-\alpha) \alpha A_{t}^{\max } L^{1-\alpha} k_{\tau}^{\alpha} d \tau,
$$

where $\lambda p\left(n_{i t}\right)$ is the flow probability that an innovation occurs in that sector.

The cost of one unit of research in terms of output is 1 . Therefore, since $n_{t}=\frac{N_{t}}{A_{t}^{\text {max }}}$, the cost of one unit of research intensity is $A_{t}^{\max }$. We assume that there exists a proportional subsidy to innovation that reduces its cost. Thus, the marginal cost of increasing research intensity is $\left(1-s_{n}\right) A_{t}^{\max }$ units of output, where $s_{n}$ is the subsidy to innovative activity. The marginal benefit of one unit of research intensity is the product of the value of the innovation $V_{t}$ and the private marginal effect of research on that sector's rate of innovation $\lambda \frac{p\left(n_{t}\right)}{n_{t}}$. Thus, the research firm sees itself as facing a constant arrival rate $\lambda \frac{p\left(n_{t}\right)}{n_{t}} n_{j t}$ per unit of research expenditure where $n_{t}$ is the sector's $\mathrm{R} \& \mathrm{D}$ expenditure and $n_{j t}$ is the firm's research intensity. Hence, the research arbitrage condition is

$$
1-s_{n}=\left(\frac{\lambda p\left(n_{t}\right)}{n_{t}}\right)\left(\frac{(1-\alpha) \alpha L^{1-\alpha} k_{t}^{\alpha}}{r_{t}+\lambda p\left(n_{t}\right)}\right) .
$$

Notice that this arbitrage condition establishes a relationship between the equilibrium values of capital and research intensity.

\subsection{Capital market}

Capital is used as a factor of production in the intermediate goods sector. We have seen that equilibrium in the capital market requires the rental rate to satisfy equation (3). The owner of a unit of capital will obtain $\zeta_{t}$ for it. This amount must be enough to cover the cost of capital. This includes the rate of interest $\left(r_{t}\right)$, the depreciation rate $(\delta)$, and the tax rate on capital accumulation $\left(\tau_{k}\right)$. Hence, the capital market arbitrage equation is

$$
r_{t}+\delta+\tau_{k}=\alpha^{2} L^{1-\alpha} k_{t}^{\alpha-1},
$$

which establishes a decreasing relationship between the interest rate and capital intensity.

\subsection{Research policy}

There exist three major types of public intervention in the research sector. The first one, already introduced in the model, consists of tax incentives to reduce the private cost of research production. In addition, the 
government may directly modify the total amount of output invested in research. We will assume that it can do so in two different ways. It can produce research at public institutions without any kind of collaboration with private firms and in direct competition with them. This policy is dubbed public provision of research. On the other hand, the government may fund private projects, acting in close collaboration with private firms. To simplify, the government is assumed to act altruistically in this case, which implies that the patent remains with the private firm. To differentiate it from the previous policy, we will refer to this one as public funding. Let us now analyze the implications for the basic model of these two types of policies.

\subsubsection{Public provision of research.}

Assume the government can perform research in the same conditions as private firms and define $\tilde{\Gamma}_{a}$ and $\tilde{\Gamma}_{b}$ as the amounts of output invested in applied and basic research by public institutions. ${ }^{6}$ Thus, public applied and basic research intensity will be given by $\Gamma_{a}=\frac{\tilde{\Gamma}_{a}}{A_{t}^{\max }}$ and $\Gamma_{b}=\frac{\tilde{\Gamma}_{b}}{A_{t}^{\max }}$. Therefore, the probability that the public sector gets an innovation will be given by $\lambda\left[\Gamma_{a}\left(1+b \Gamma_{b}\right)\right]^{\frac{1}{2}}$. The additional research implies that the total probability of an innovation occurring in a given sector will now be $\lambda\left[n_{a}\left(1+b n_{b}\right)\right]^{\frac{1}{2}}+\lambda\left[\Gamma_{a}\left(1+b \Gamma_{b}\right)\right]^{\frac{1}{2}}$. Consequently, the research arbitrage equation will be given by

$$
1-s_{n}=\left(\frac{\lambda p(n)}{n}\right)\left(\frac{(1-\alpha) \alpha L^{1-\alpha} k_{t}^{\alpha}}{r_{t}+\lambda p(n)+\lambda\left[\Gamma_{a}\left(1+b \Gamma_{b}\right)\right]^{\frac{1}{2}}}\right) .
$$

Notice that public research in this case induces a higher rate of creative destruction, i.e. a higher probability that the owner of the patent is replaced. Therefore, the research activity of the public sector reduces the present value of an innovation for a private researcher. In the case that the public sector gets the patent, it will be sold to an intermediate good producer and the value of the patent will be transferred to consumers in the form of a lump sum transfer.

\subsubsection{Public funding of research.}

This type of research policy directly affects the microeconomic decision of the research firm about the amounts to be invested in basic and applied research. Consequently, we must rewrite the problem of the firm as follows:

$$
\max _{n_{a}, n_{b}} \lambda\left[\left(n_{a}+\Gamma_{a}\right)\left(1+b\left(n_{b}+\Gamma_{b}\right)\right)\right]^{\frac{1}{2}},
$$

subject to the following constraints:

$$
\begin{aligned}
n_{i t} & =n_{a}+n_{b} \\
n_{a} & \geq 0 \\
n_{b} & \geq 0 .
\end{aligned}
$$

${ }^{6}$ We assume that the amount invested in each sector is the same so that aggregate and sectoral amounts coincide. 
The optimal choices for $n_{a}$ and $n_{b}$ are

$$
\begin{aligned}
& n_{a}= \begin{cases}\frac{n_{i t}+\Gamma_{b}-\Gamma_{a}}{2}+\frac{1}{2 b} & \text { if } n_{i t}+\Gamma_{a}-\Gamma_{b} \geq \frac{1}{b} \\
n_{i t} & \text { otherwise },\end{cases} \\
& n_{b}= \begin{cases}\frac{n_{i t}+\Gamma_{a}-\Gamma_{b}}{2}-\frac{1}{2 b} & \text { if } n_{i t}+\Gamma_{a}-\Gamma_{b} \geq \frac{1}{b} \\
0 & \text { otherwise. }\end{cases}
\end{aligned}
$$

We will consider only situations with $n_{b}$ positive in the main text. The results when private firms do not perform basic research may be found in Appendix D. For $n_{a}$ and $n_{b}$ positive, the probability of obtaining an innovation given $n_{i t}$ is

$$
\lambda p\left(n_{i t}, \Gamma\right)=\frac{\lambda\left[1+b\left(n_{i t}+\Gamma\right)\right]}{2 \sqrt{b}},
$$

where $\Gamma=\Gamma_{a}+\Gamma_{b}$. Again, the symmetric behavior of the sectors in equilibrium allows us to drop the $i$ subindex of $n_{i t}$ in (11). The probability of the project being successful per unit of research intensity is thus

$$
\frac{\lambda p\left(n_{t}, \Gamma\right)}{n_{t}} .
$$

Therefore, the research arbitrage equation is given by

$$
1-s_{n}=\left(\frac{\lambda p\left(n_{t}, \Gamma\right)}{n_{t}}\right)\left(\frac{(1-\alpha) \alpha L^{1-\alpha} k_{t}^{\alpha}}{r_{t}+\lambda p\left(n_{t}, \Gamma\right)}\right) .
$$

The main difference with the previous policy in terms of the implications for the research arbitrage equation, is that even though the effect on the rate of creative destruction still remains, there is an additional effect on the productivity of research. This effect is represented by the fact that in the presence of public funding, the probability of obtaining an innovation per unit of private research intensity is now given by $\frac{\lambda(1+b(n+\Gamma))}{2 \sqrt{b} n}$ rather than by $\frac{\lambda(1+b n)}{2 \sqrt{b} n}$.

\subsection{Equilibrium}

General equilibrium is defined by the two equations determining equilibrium in the capital and research sectors. These equations are

$$
r_{t}+\delta+\tau_{k}=\alpha^{2} L^{1-\alpha} k_{t}{ }^{\alpha-1}
$$

for the capital market and

$$
1-s_{n}=\left(\frac{\lambda p\left(n_{t}\right)}{n_{t}}\right)\left(\frac{(1-\alpha) \alpha L^{1-\alpha} k_{t}^{\alpha}}{r_{t}+\lambda p\left(n_{t}\right)+\lambda\left[\Gamma_{a}\left(1+b \Gamma_{b}\right)\right]^{\frac{1}{2}}}\right),
$$

for the research market in the case of public provision or,

$$
1-s_{n}=\left(\frac{\lambda p\left(n_{t}, \Gamma\right)}{n_{t}}\right)\left(\frac{(1-\alpha) \alpha L^{1-\alpha} k_{t}^{\alpha}}{r_{t}+\lambda p\left(n_{t}, \Gamma\right)}\right)
$$


for the case of public funding.

The systems formed by equations (12) and (13), and (12) and (14) define the equilibrium values for $k_{t}$ and $n_{t}$ in each case. These systems implicitly determine a relationship between capital and research intensity that allows us to analyze the dynamics of the model in terms of capital and consumption. The laws of motion for capital and consumption are given by

$$
\dot{K}_{t}=Y_{t}-C_{t}-N_{t}-\delta K_{t},
$$

and

$$
\dot{C}_{t}=\left(r_{t}-\rho\right) C_{t}
$$

where (15) is derived from the consumer's optimization problem. These expressions can be written in efficiency units as follows:

$$
\begin{aligned}
\dot{k_{t}} & =L^{1-\alpha} k_{t}^{\alpha}-c_{t}-\frac{1}{E(a)} n_{t}-\left(\delta+\gamma_{t}\right) k_{t} \\
\dot{c_{t}} & =\left(r_{t}-\rho-\gamma_{t}\right) c_{t},
\end{aligned}
$$

where $\gamma_{t}$ is the growth rate of $A_{t}^{\max }$ and therefore is given by $\sigma \lambda\left(n_{A}\right)^{\beta}\left(n_{B}\right)^{1-\beta}$ which, ultimately is a continuous function of $n_{t}$. Let $n^{d}\left(k_{t}\right)$ be the dynamic relationship between capital and research intensity defined by equations (12) and (13) or (12) and (14). ${ }^{7}$ Then, we can express equations (16) and (17) in terms of $k_{t}$ and $c_{t}$ exclusively

$$
\begin{aligned}
\dot{k_{t}} & =L^{1-\alpha} k_{t}^{\alpha}-c_{t}-\frac{1}{E(a)} n^{d}\left(k_{t}\right)-\left(\delta+\gamma^{d}\left(k_{t}\right)\right) k_{t} \\
\dot{c_{t}} & =\left(\alpha^{2} L^{1-\alpha} k_{t}^{\alpha-1}-\delta-\tau_{k}-\rho-\gamma^{d}\left(k_{t}\right)\right) c_{t} .
\end{aligned}
$$

Due to the non-linearity of the system we proceed with its linearization around the steady state in order to analyze the dynamics of the model. It can be proved that the system exhibits local saddle path stability around the steady state. Therefore, we can perform comparative statics analysis at the long run equilibrium.

\section{Steady state}

In equilibrium the production function is simplified due to the fact that the equilibrium value of intermediate input is the same for every sector. Consequently, we may write equation (2) as

$$
Y_{t}=A_{t} L^{1-\alpha} k_{t}^{\alpha},
$$

\footnotetext{
${ }^{7}$ Specifically, $n^{d}\left(k_{t}\right)$ is obtained as follows: equation (12) defines the interest rate as a function of $k_{t}$. Therefore, we can substitute in either (13) or (14) so as to obtain $n_{t}$ as an implicit function of capital intensity. Depending on whether we are considering public provision or direct funding, $n^{d}\left(k_{t}\right)$ is defined by equation (13) or (14). Consequently, we should use a different notation for each function. However, for the sake of simplicity and because the implications for the dynamics of the model are equivalent, we denote the two functions by $n^{d}\left(k_{t}\right)$.
} 
which implies that in a steady state, the rate of growth of output will be the rate of growth of aggregate productivity. That is

$$
\gamma=\sigma \lambda\left(n_{A}\right)^{\beta}\left(n_{B}\right)^{1-\beta}
$$

Using this result, and the fact that in a steady state $k$ and $n$ are constant we may write equations (12), (13) and (14) as follows:

$$
\begin{gathered}
\gamma+\rho+\delta+\tau_{k}=\alpha^{2} L^{1-\alpha} k^{\alpha-1} \\
1-s_{n}=\left(\frac{\lambda p(n)}{n}\right)\left(\frac{(1-\alpha) \alpha L^{1-\alpha} k^{\alpha}}{\gamma+\rho+\lambda p(n)+\lambda\left[\Gamma_{a}\left(1+b \Gamma_{b}\right)\right]^{\frac{1}{2}}}\right), \\
1-s_{n}=\left(\frac{\lambda p(n, \Gamma)}{n}\right)\left(\frac{(1-\alpha) \alpha L^{1-\alpha} k^{\alpha}}{\gamma+\rho+\lambda p(n, \Gamma)}\right) .
\end{gathered}
$$

Equations (21) and (22) on one hand and (21) and (23) on the other determine the steady state values of $k$ and $n$ for the two alternative assumptions. Let us consider the two research policies separately.

\subsection{Public provision}

If research is performed at public institutions, in direct competition with private firms, the equations determining $k$ and $n$ are (21) and (22). The growth effect of giving tax incentives to private research firms is established in the following proposition:

Proposition 1 The long run growth rate increases when the subsidy rate to private research is raised.

Proof. See Appendix B.1.

The cost reduction induced by the subsidy increases the optimal choice of private research intensity. The higher investment in research implies a larger productivity growth and hence, the economy will grow faster.

Concerning the effect of public provision of research, notice that if no public applied research is performed, the amount of basic research produced at public institutions does not affect the rate of creative destruction. However, this research adds to the stock of knowledge and will make private research more productive via spillovers, both of the intertemporal and intersectoral varieties. Therefore, the effect on growth of increasing public investment in basic research should be positive. However, a higher value of $\Gamma_{b}$ will reduce private research intensity. This crowding out of private research is due to the increase in factor prices induced by the higher public investment. Nevertheless, the reduction in private research is not large enough to compensate for the positive effect of the public investment and the final net result on the growth rate is positive. On the contrary, if public applied research is positive then basic research has an additional effect. Namely, that it will increase the probability that the public sector gains a patent. This will induce a larger crowding out of private research and reduce the expected life of any future innovation, because the rate of replacement will 
be higher. Whether the final impact on growth will be positive or negative depends upon the levels of public basic and applied research and on the parameter values. Due to the ambiguity in the sign of the growth effect we proceed to define parameter subspaces for which the growth derivative shows the desired sign. Let us define the following vectors of parameters: $\theta \equiv\left(\alpha, \delta, \rho, \lambda, s_{n}, \tau_{k}, \sigma, L\right) \in \Theta$ where $\Theta \equiv[0,1]^{6} \times(0, \infty)^{2}$, $\psi \equiv\left(\beta, b, \Gamma_{a}, \Gamma_{b}\right) \in \Psi$ where $\Psi \equiv[0,1] \times[0, \infty)^{3}$ and $\omega \equiv \theta \times \psi \in \Theta \times \Psi$. Denote the parameter space by $\Omega \equiv \Theta \times \Psi$ and define the following subspaces of $\Psi$ and $\Omega$ :

$$
\begin{gathered}
\Psi_{1}=\left\{\psi \in \Psi \mid \text { either } \frac{1}{2(1-\beta)}\left(\frac{b \Gamma_{a}}{1+b \Gamma_{b}}\right)^{\frac{1}{2}}<1 \text { and } 1+b\left(\Gamma_{a}-\Gamma_{b}\right)>0\right. \\
\text { or } \left.\frac{1}{2}\left(\frac{b \Gamma_{a}}{1+b \Gamma_{b}}\right)^{\frac{1}{2}}\left(1+\left(\frac{\beta}{1-\beta}\right) \frac{b \Gamma_{b}}{1+b \Gamma_{a}}\right)<1 \text { and } 1+b\left(\Gamma_{a}-\Gamma_{b}\right)<0\right\}, \\
\Psi_{2}=\left\{\psi \in \Psi \mid \text { either } \frac{1}{2 \beta}\left(\frac{1+b \Gamma_{b}}{b \Gamma_{a}}\right)^{\frac{1}{2}}<1, \Gamma_{a}>0 \text { and } 1+b\left(\Gamma_{a}-\Gamma_{b}\right)<0\right. \\
\text { or } \left.\frac{1}{2}\left(\frac{1+b \Gamma_{b}}{b \Gamma_{a}}\right)^{\frac{1}{2}}\left(1+\left(\frac{1-\beta}{\beta}\right) \frac{1+b \Gamma_{a}}{b \Gamma_{b}}\right)<1, \Gamma_{a}>0 \text { and } 1+b\left(\Gamma_{a}-\Gamma_{b}\right)>0\right\}, \\
\Omega_{1}=\left\{\omega \in \Omega \mid \frac{1}{2}\left(\frac{b \Gamma_{a}}{1+b \Gamma_{b}}\right)^{\frac{1}{2}}>1+\epsilon, \chi_{1}>\chi_{2} \text { and } \chi_{3}<\chi_{4}\right\},
\end{gathered}
$$

and

$$
\Omega_{2}=\left\{\omega \in \Omega \mid \frac{1}{2}\left(\frac{1+b \Gamma_{b}}{b \Gamma_{a}}\right)^{\frac{1}{2}}>1+\epsilon, \chi_{1}>\chi_{2} \text { and } \chi_{3}<\chi_{4}\right\}
$$

where $\epsilon, \chi_{1}, \chi_{2}, \chi_{3}$ and $\chi_{4}$ are defined in Appendix B.1. The following propositions establish the effect of public basic and applied research on growth:

Proposition 2 If either $\Gamma_{a}=0$ or $\psi \in \Psi_{1}$ then, the effect on growth of public basic research is positive. Conversely, if $\omega \in \Omega_{1}$, the growth effect of $\Gamma_{b}$ is negative.

\section{Proof. See Appendix B.1.}

Proposition 2 implies that the effect on growth of public basic research is ambiguous when there exists a positive level of public applied research. Intuitively, a larger public investment in basic research will make the economy grow faster when the existing level of public applied research is not too large and $\Gamma_{b}$ keeps in a certain range relative to $\Gamma_{a}$. On the contrary, in order to find a negative effect on growth, the amount of public applied research must be very large relative to the amount of public basic research. In any case, when both $\Gamma_{a}$ and $\Gamma_{b}$ are very large, the effect on growth will generally be positive, due to the fact that for high levels of public research intensity, the level of private research will be so low that the relevant variables for the growth rate of the economy will be the amounts of public investment.

Proposition 3 If $\psi \in \Psi_{2}$ then the effect on growth of public applied research is positive. On the contrary, if $\omega \in \Omega_{2}$ then $\frac{d \gamma}{d \Gamma_{a}}<0$. 


\section{Proof. See Appendix B.1.}

If the public sector decides to increase public applied research from zero, the most relevant effect will be a large crowding out of private research. As a consequence, the rate of growth of the economy will generally fall when the levels of public applied research are close to zero. However, if public investment in applied research keeps growing the effect on growth may be inverted. This is so because the crowding out of private research is smaller as $\Gamma_{a}$ grows. The conditions in Proposition 3 require large values of both basic and applied public research in order to have a positive effect on growth of $\Gamma_{a}$ and small values of public applied research or large differences between basic and applied investments in order to have a negative effect on growth.

In summary, what the previous propositions require is that the amounts invested in applied and basic research keep certain proportions. If the investment in one of the two types of research is too large or too small relative to the other then the effect on growth will be negative. Accordingly, it appears interesting to analyze the implications of public R\&D when it is divided into basic and applied research following a certain rule. Given that this type of public intervention depicts the public sector behaving as a private research firm, we want to consider also the effect of public research if the amounts of public basic and applied research are chosen so as to get the maximum probability of obtaining an innovation for a given amount of public investment in research. In other words, let $\Gamma=\Gamma_{a}+\Gamma_{b}, \Gamma_{a}=\frac{1+b \Gamma}{2 b}$ and $\Gamma_{b}=\frac{b \Gamma-1}{2 b}$ for $\Gamma \geq \frac{1}{b}$. Then, $\left(\Gamma_{a}\left(1+b \Gamma_{b}\right)\right)^{\frac{1}{2}}=p(\Gamma)$ and the comparative statics results of marginal changes in $\Gamma$ are as follows:

Proposition 4 The effect on the steady state growth rate of a marginal increase in $\Gamma$ is positive.

Proof. See Appendix B.1.

The result established in the previous proposition implies that the public sector can actually substitute the private research sector and, since we have assumed the same productivity for the private and the public sector, this would be beneficial for the growth performance of the economy. However, this result is due to the assumption that the amount of research invested by the public sector is not limited by profitability conditions, since it may be financed by lump sum taxes. If we assumed instead that the public sector must look for finance in the creditl market, then it would be constrained by the same research arbitrage equation as private firms, and there would exist a maximum level of research at which its marginal cost equals the marginal benefit.

\subsection{Public funding}

If research policy consists on the provision of funds for private firms' research projects, the relevant equations in order to determine the steady state values of $n$ and $k$ are (21) and (23). In this case, the following propositions apply:

Proposition 5 A higher subsidy rate to private research increases the steady state growth rate of the economy. 


\section{Proof. See Appendix B.2.}

Proposition 5 shows that the effect of a subsidy to private research is not affected by the assumption on whether public research is performed at public institutions or in coordination with private firms. Thus, the concession of tax incentives to private research continues having a positive effect on long run growth, since it increases the amount of private research intensity. Notice also that an increase in $n$ reduces the ratio of total applied research to total basic research. This may suggest that the privately chosen amounts of applied and basic research are biased towards applied research, while the economy could benefit from a reduction of this ratio. With respect to the effects of the amounts devoted to public research, we find that they are quite different to public provision, as the next proposition establishes:

Proposition 6 A higher research intensity in either applied or basic fields implies a larger rate of growth in the long run. In equilibrium, the effects of marginal changes of applied and basic research on the growth rate are identical.

\section{Proof. See Appendix B.2.}

The positive effect on private research productivity of this type of research policy outweighs the negative effect of the higher probability of replacement induced by public research, which makes the crowding out of private R\&D smaller or even, in some cases cause the opposite effect. That is, we can find situations in which an increase of public research implies a higher amount of private $R \& D$ investment. The effect on private research is thus ambiguous, as opposed to the previous case, in which private R\&D always decreases after an increase in public research. Concerning the result that the effects of public applied and basic research are identical in terms of growth, it is due to the fact that private firms internalize the funds provided by the public sector in such a way that if for instance, the amount of public basic research is increased, the firm will reduce its own investment in basic research and devote more resources to applied research. The same applies for public applied research. Therefore, the behavior of the firm neutralizes the possibility of having different effects on growth of these two types of public R\&D. In addition, we find that an increase in either applied or basic public research is going to reduce the ratio of total applied research to total basic research and from Proposition 6 we know that this is going to have a positive effect on growth. Thus, we find again, as in the case of research subsidies, that a reduction of $\frac{n_{A}}{n_{B}}$, with $n_{A}$ and $n_{B}$ increasing, is beneficial for the rate of growth of the economy.

One of the main differences between public funding of research and direct R\&D subsidies is that with public funding the government may choose the amounts devoted to basic and applied fields. The result established in Proposition 6 indicates that this difference will not be relevant for the growth performance of the economy. However, this does not imply that both policies are equivalent. If we want to compare the growth effects of research subsidies and public research, we can take as reference the no intervention equilibrium and compare the growth and private research derivatives with respect to the policy instruments. The following propositions compare the effects of the introduction of these policies: 
Proposition 7 If $s_{n}=0$ and $\Gamma=0$, then the growth effects of equivalent changes in public funding of research and the research subsidy are equal.

Corollary 8 If $s_{n}=0$ and $\Gamma=0$, then

$$
\frac{d n}{d \Gamma}=\frac{d n}{d s_{n}}\left(\frac{1}{n}\right)-1
$$

Proofs. See Appendix B.2.

The previous results compare the effects of the two policies at $s_{n}=0$ and $\Gamma=0$ because at this point they can be made equivalent in terms of the public budget. Two policies are equivalent in terms of the public budget if they imply the same fiscal effort. ${ }^{8}$ Thus, if the government's budget is given by

$$
T_{t}=s_{n} N_{t}+\Gamma A_{t}^{\max }-\tau_{k} K_{t},
$$

any two policies that we wish to compare must imply the same marginal change in the lump sum tax $T_{t}$ used to balance the budget. We find that the growth effects are identical, however the effects on private research differ, since the subsidy will always induce a larger increase in this variable. Intuitively, the research subsidy provokes a larger investment from the private sector, while the increase in public funding provides an extra investment that allows the private sector to reduce their investment effort. Therefore, even though their effect on growth is equivalent, they have different effects on research intensity and probably on consumption and welfare. The choice of policy will thus depend on how the authorities want to influence private research investment.

In summary, we find that both tax incentives and public funding of private research have unambiguously positive effects on long run growth and therefore, are research policies that can be undertaken without fear of damaging the growth performance of the economy. However, public provision of research is a more dangerous tool, since under some conditions, public research can be harmful for the private R\&D sector and the economy as a whole. Nevertheless, if public provision of research were exclusively confined to basic fields, or if basic and applied research are kept in the right proportions, the negative effects of this type of policy would be avoided.

Concerning the debate on whether public research should be more market oriented or be devoted only to the accumulation of basic knowledge, the model predicts different results depending on which specific policy is carried over. If we are considering public funding of private research and we take funds from basic research to use them in applied fields, the effect on long run growth will be null due to the accommodating behavior of private research firms. However, in the case of public provision, a redirection of funds from basic to applied fields will have positive or negative effects depending on the initial situation of the economy.

\footnotetext{
${ }^{8}$ See the proof of Proposition 7 for the adjustment necessary to make the changes in the instruments equivalent in terms of the public budget.
} 


\section{Welfare analysis}

From equation (1) we can express utility at the steady state in terms of the stationary level of consumption and the long-run growth rate

$$
V_{s}(c, \gamma)=\int_{0}^{\infty} \ln \left(c A_{t}\right) e^{-\rho t} d t=\frac{\ln \left(c A_{0}\right)}{\rho}+\frac{\gamma}{\rho^{2}}
$$

The change in steady state welfare is a combination of the change in steady state consumption and the change in steady state growth

$$
\frac{\partial V_{s}(c, \gamma)}{\partial x}=\frac{1}{\rho c} \frac{\partial c}{\partial x}+\frac{1}{\rho^{2}} \frac{\partial \gamma}{\partial x}
$$

where $x$ represents any of the three policy instruments, $s_{n}, \Gamma_{a}$ and $\Gamma_{b}$.

This measure of welfare is valid to compare two situations of long run equilibrium. However, it does not consider the periods of transition during which the economy moves from one equilibrium to another. In order to reflect the transition we must analyze the effect on lifetime utility. Rewrite equation (1) to obtain the following expression for lifetime utility:

$$
V(x)=\frac{\ln \left(A_{0}\right)}{\rho}+\int_{0}^{\infty}\left[\int_{0}^{t} \gamma_{s}(x) d s\right] e^{-\rho t} d t+\int_{0}^{\infty} \ln \left(c_{t}(x)\right) e^{-\rho t} d t
$$

where $\gamma_{t}(x)$ and $c_{t}(x)$ are the time paths of the growth rate and the level of consumption per efficiency unit after a change in one of the policy parameters. The effect on utility will thus be given by the effects on the paths of growth and consumption. I will obtain first the effect on the paths of consumption and capital intensity and then use the latter to get the effect on the path of the growth rate.

Let $c=p(k, x)$ be the saddle path of the system which can be interpreted as the graph of a policy function relating consumption and capital. Then we know that its slope, $p_{k}$ its positive and equal to $\frac{\phi_{k}}{\lambda_{1}} \cdot{ }^{9}$ Substituting the policy function into the law of motion of capital, which we denote by $\varphi(k, c)$, the equilibrium dynamics of the system can be characterized by a single differential equation which describes the evolution of the state variable along the stable manifold.

$$
\dot{k}=\varphi(k, c)=\varphi(k, p(k, x))=\Psi(k, x) .
$$

The solution to this equation, $k_{t}(x)$, gives the equilibrium value of $k$ as a function of time and the policy parameter. Using $k_{t}(x)$ in the policy function we would obtain the time path of $c$

$$
c_{t}(x)=p\left(k_{t}(x), x\right)
$$

To calculate the change in welfare we need the derivative of the whole time path of $c$ with respect to $x$

$$
\frac{d c_{t}(x)}{d x}=p_{k} \frac{d k_{t}(x)}{d x}+p_{x}
$$

\footnotetext{
${ }^{9}$ We denote by $\phi_{k}$ the derivative with respect to capital of the law of motion for consumption evaluated at the steady state, and $\lambda_{1}$ is the negative eigenvalue of the system formed by (18) and (19) also evaluated at the steady state.
} 
where $p_{x}$ is the derivative of the policy function with respect to the policy instrument or graphically, the shift in the saddle path caused by the policy change.

In order to compute $\frac{d k_{t}(x)}{d x}$, notice that $k_{t}(x)=k(t, x)$ must satisfy identically the original equation

$$
\dot{k}(t, x) \equiv \varphi(p(k(t, x), x), k(t, x), x),
$$

where $\varphi(c, k ; x)$ is the law of motion of capital given by equation (18). Differentiate both sides with respect to $x$

$$
\dot{k}_{x}=\frac{d k_{x}}{d t}=\left[\varphi_{c} p_{k}+\varphi_{k}\right] k_{x}+\varphi_{c} p_{x}+\varphi_{x}
$$

Hence $k_{x}$ satisfies a linear differential equation. Moreover, when we start from a steady state, the coefficients of this equation are constant and we can write

$$
\dot{k}_{x}=\lambda_{1} k_{x}-p_{x}+\varphi_{x}
$$

The general solution is given by

$$
k_{x}(t)=\exp \left(\lambda_{1} t\right) k_{x}(0)+\left(1-\exp \left(\lambda_{1} t\right)\right) k_{x}(\infty) .
$$

Since $k$ is a predetermined variable, the change at the date of the policy change $k_{x}(0)$ must be zero. The long run effect, $k_{x}(\infty)=\lim _{t \rightarrow \infty} k_{x}(t)$, is in fact the derivative of the steady state value of $k$ with respect to the policy parameter, and can be expressed as

$$
k_{x}(\infty)=\frac{p_{x}-\varphi_{x}}{\lambda_{1}}
$$

The equilibrium time path of the derivative of $k$ with respect to $x$ is thus given by

$$
k_{x}(t)=\left(1-\exp \left(\lambda_{1} t\right)\right)\left[\frac{p_{x}-\varphi_{x}}{\lambda_{1}}\right],
$$

that is, $k$ will gradually reach its new steady state value at a rate equal to the negative eigenvalue.

Substitute now in equation (25) to obtain the final expression for the derivative of the time path of consumption with respect to the policy parameter

$$
\frac{d c_{t}(x)}{d x}=p_{k}\left(1-\exp \left(\lambda_{1} t\right)\right)\left[\frac{p_{x}-\varphi_{x}}{\lambda_{1}}\right]+p_{x} .
$$

As before, we can identify the immediate change and the long run effect

$$
\begin{aligned}
\frac{d c_{0}(x)}{d x} & =p_{x} \\
\frac{d c_{\infty}(x)}{d x} & =p_{k}\left[\frac{p_{x}-\varphi_{x}}{\lambda_{1}}\right]+p_{x}
\end{aligned}
$$

where the first represents the necessary jump of consumption to get on the new saddle path and the second is the effect on the steady state value of consumption. Thus, consumption will initially jump to the new saddle path and then it will approach its new steady state value at a rate equal to $\lambda_{1}$. 
The derivative of the growth rate and consumption per efficiency unit at date $t$ are given by ${ }^{10}$

$$
\begin{aligned}
\frac{d \gamma_{t}(x)}{d x} & =\frac{d \gamma^{d}(k)}{d k}\left(1-\exp \left(\lambda_{1} t\right)\right) \frac{d k}{d x}+\frac{d \gamma^{d}(k)}{d x} \\
\frac{d c_{t}(x)}{d x} & =\frac{d c}{d x}-p_{k} \exp \left(\lambda_{1} t\right) \frac{d k}{d x} .
\end{aligned}
$$

Hence, the change in welfare will be given by the following expression:

$$
\frac{d V(x)}{d x}=\frac{d V_{s}(x)}{d x}+\left[\frac{\left(\frac{\rho-\lambda_{1}}{\rho}\right) \frac{d \gamma^{d}(k)}{d k}+\frac{(1-\alpha) \zeta}{k}}{\lambda_{1}\left(\rho-\lambda_{1}\right)}\right] \frac{d k}{d x} .
$$

Equations (24) and (26) give the general expressions for the effect of the three policies on the different measures of welfare. Given that the expression in square brackets is negative, the relationship between the two measures of welfare will be determined by the sign of $\frac{\partial k}{\partial x}$ in each case.

Consider the change in welfare excluding the periods of transition, that is, equation (24). If steady state consumption and growth evolve in opposite directions, the actual value of the discount rate $\rho$ will be determinant for the sign of the welfare change and we will not be able to give an unambiguous sign to the change in welfare without assuming a specific value for the discount rate. Unfortunately, this will normally be the case. Just for illustrative purposes, a calibration was made for empirically acceptable values of the parameters. ${ }^{11}$ Table 1 suggests that the research subsidy may have positive effects on welfare though only for low values of the policy instrument. However, if we introduce a slight change in the parameters, the range of values for which an increase of $s_{n}$ is welfare improving is substantially enlarged. In summary thus, the calibration is suggesting that the research subsidy may increase welfare, though for high values of $s_{n}$ the change in welfare becomes negative. Figure 1 illustrates the effect on the two measures of welfare taking as reference the level of welfare attained at $s_{n}=0$.

Regarding the effects on consumption of public research, we cannot give an unambiguous sign to the relevant derivatives, which implies that very little can be said about the effect on welfare of policies affecting the level of public research. Nevertheless, the simulation results presented in tables 1 to 7 suggest that the effect on welfare of marginally increasing public R\&D may be positive. In particular, Tables 2 and 3 present the effect on consumption and welfare of marginal changes in public basic research. If public applied research is zero, a marginal increase in basic $R \& D$ is negative for steady state consumption. However, the effect on the measure of welfare that includes the periods of transition is initially positive. In addition, we found that this result is quite robust to changes in parameters other than the discount rate. Figure 2 shows the relationship between welfare and public basic research. Observe that as the amount of basic research increases its effect on the growth rate diminishes while consumption per efficiency unit decreases due to the taxes necessary to finance this policy. As a consequence, the effect on welfare becomes negative for high values of public R\&D investment. Table 3 shows the results when public applied research is positive. Notice

\footnotetext{
${ }^{10}$ The derivatives of $\gamma^{d}$ are evaluated at the steady state because we consider the stationary equilibrium as the situation before the tax change.

${ }^{11}$ Refer to Appendix C for the calibration results.
} 
that the introduction of basic research reduces the growth rate, implying an initial reduction of the measure of welfare that includes the periods of transition. Further increases in $\Gamma_{b}$ will make the economy grow faster as we move into the set of policy parameter combinations that increases $\gamma$. Nevertheless, the two measures of welfare fall for these higher values of public basic research as shown in Figure 3. Similarly, Table 4 shows the results obtained for changes in public applied research. They show that there exists a range of values of $\Gamma_{a}$ for which the effect on welfare may be positive. The results obtained for the case in which the public sector chooses the amounts of basic and applied research as if it were a private firm (table 5) are very similar and show a remarkable crowding out of private research.

The results under the public funding assumption, presented in tables 6 and 7, and Figures 6 and 7 indicate that the effect on welfare of research subsidies and public R\&D may be positive but only for low values of the policy parameters.

\section{Conclusions}

This paper has addressed the issue of the need for an active research policy and has discussed the implications of the different alternatives that actual governments have at their disposal. The analysis has been performed in the context of an endogenous growth model with technological change in which private firms may invest both in applied and basic research. The difference between these two types of research is relevant due to the existing debate on whether public research should limit itself to basic knowledge or, on the contrary, should be directed to projects with market applications. In addition, it has been found that subsidies to private research will make the economy grow faster and may increase consumer welfare since this policy increases private research investment.

Public research performed at public institutions has different implications depending on whether this research is directed towards basic or applied fields. When public research is exclusively concerned with basic knowledge, the effect on growth and welfare of this type of public investment is positive. This is due to the fact that innovations arising from public basic research will add to the stock of knowledge and spillover to the rest of the economy. These spillovers translate into more important technological improvements when private $\mathrm{R} \& \mathrm{D}$ is successful, which determines a higher growth rate of aggregate technology and hence, of the economy. However, if public institutions do research also in applied fields, any innovation arising from this type of research will be patentable. This implies that public institutions will compete with private firms in the patent race and thus, public research will have to be included when considering the rate of replacement of a sector. This rate is given by the probability that an innovation occurs in a given sector and determines the expected life of an existing patent. The effect of public applied research on the rate of replacement represents a negative externality for private research firms, since the expected value of an innovation falls when the rate of replacement increases. However, public applied research also adds to the stock of knowledge and in consequence, causes a positive external effect. The interaction of these opposing forces determines an ambiguous effect of public applied research on growth. 
On the other hand, we have found that public funding of private projects has an unambiguously positive growth effect. This is mainly due to the higher productivity of private research induced by this policy and to the fact that it does not necessarily crowd out private research. Indeed, whether public funding of research projects induces more private investment or not depends upon the initial situation of the economy and on the actual values of the parameters though, for a set of plausible parameter values, it is easy to obtain the result that private and public research behave as complements rather than substitutes. We observe that in equilibrium, the funding of either applied or basic projects have identical effects on private research and growth. Therefore, if research policy is instrumented through the funding of private projects, it is irrelevant whether the funds are used for basic or applied projects. This is turn implies that moving funds from basic projects to more applied fields, as proposed by the "new economic instrumentalism", ${ }^{12}$ would have a null effect on long run growth. Additionally, we have compared the effects on growth of subsidizing research as opposed to public funding of projects. We have found that the effect on growth of both policies is equal when evaluated at the no intervention equilibrium.

\section{References}

[1] Aghion, P. and P. Howitt (1998) "Endogenous growth theory" MIT Press.

[2] Aghion, P. and P. Howitt (1992) "A model of growth through creative destruction" Econometrica LX, 323-351.

[3] Aghion, P. and J. Tirole (1994) "Opening the black box of innovation" European Economic Review 38, 701-710.

[4] Aghion, P. and J. Tirole (1994) "The management of innovation" The Quarterly Journal of Economics 109, 1185-1209.

[5] Cassiman, B., Pérez Castrillo, D., Veugelers, R. (2001) "Endogenizing know-how flows through the nature of R\&D investments." CEPR Discussion Paper 2622.

[6] David, P. A. (2000) "The political economy of public science" in Smith, H.L. (ed), "The Regulation of Science and Technology" MacMillan, London.

[7] David, P. A., Hall, B. H., Toole, A. A. (2000) "Is public R\&D a complement or a substitute for private R\&D? A review of the econometric evidence." in Link, A. N. (ed) Research Policy 29 (4-5), 497-529 Special Millennium Issue.

[8] David, P. A. and Hall, B. H. (2000) "Heart of Darkness: Modelling Public-Private funding Interactions Inside the R\&D Black Box" Research Policy 29, 1165-1183.

\footnotetext{
${ }^{12}$ See David (2000).
} 
[9] De la Fuente, A. (2000) "Mathematical Methods and Models for Economists". Cambridge University Press.

[10] Delbono, F. and V. Denicolò (1993) "Regulating innovative activity. The role of a public firm." International Journal of Industrial Organization 11, 35-48.

[11] Glomm, G. and B. Ravikumar (1994) "Growth-inequality trade-offs in a model with public sector R\&D" Canadian Journal of Economics XXVII, 2, 484-493.

[12] Griliches, Z. (1986) "Productivity, R\&D, and basic research at the firm level in the 1970's" The American Economic Review 76(1), 141-154.

[13] Grossman, G. and Helpman, E. (1991a) "Quality ladders in the theory of growth" Review of Economic Studies 58, 43-61.

[14] Grossman, G. and Helpman, E. (1991b) "Innovation and growth in the global economy" Cambridge, Mass. MIT Press.

[15] Ham, R. and D. Mowery, (1998) "Improving the effectiveness of public-private R\&D collaboration: case studies at a US weapons laboratory" Research Policy 26, 661-675.

[16] Howitt, P. (1999) "Steady endogenous growth with population and R\&D inputs growing" Journal of Political Economy 107, 715-730.

[17] Howitt, P. and P. Aghion (1998) "Capital Accumulation and Innovation as Complementary Factors in Long-Run Growth" Journal of Economic Growth 3, 111-130.

[18] Mamuneas, T. (1999) "Spillovers from publicly financed R\&D capital in high-tech industries" International Journal of Industrial Organization 17, 215-239.

[19] Mamuneas, T. and M. Nadiri (1996) "Public R\&D policies and cost behavior of the US manufacturing industries" Journal of Public Economics 63, 57-81.

[20] Mansfield, E. (1995) "Academic research underlying industrial innovations: sources, characteristics and financing." The Review of Economics and Statistics 77, 55-65.

[21] Park, W. G. (1998) "A Theoretical Model of Government Research and Growth" Journal of Economic Behavior and Organization 34, 69-85.

[22] Pelloni, A. (1997) "Public financing of education and research in a model of endogenous growth" Labor 11(3), 517-539.

[23] Romer, P. (1990) "Endogenous technological change" Journal of Political Economy 98(5), s71-s102.

[24] Schumpeter, J. A. (1911) "The theory of economic development" Cambridge Ma. Harvard U. Press. 
[25] Stenbacka, R. and M. Tomback (1998) "Technology policy and the organization of R\&D" Journal of Economic Behavior and Organization 36, 503-520.

\section{A Distribution of relative productivities across sectors}

Let $F(\cdot, t)$ be the cumulative distribution function of absolute productivity coefficients $A_{i t}$ at any given date $t$. Define $\Phi(t)=F\left(A_{t_{0}}^{\max }, t\right)$. Then, $\Phi(t)$ gives us the mass of sectors with a productivity coefficient below $A_{t_{0}}^{\max }$ at date $t$. Therefore,

$$
\begin{aligned}
\Phi\left(t_{0}\right) & =1 \\
\frac{d \Phi(t)}{d t} & =-\Phi(t) d(t),
\end{aligned}
$$

where $d(t)$ is the probability that a sector innovates. Thus, depending on the assumption that we are considering, it will be given by $\lambda p\left(n_{t}\right)+\lambda \Gamma_{a}\left(1+b \Gamma_{b}\right)$ in the case of public provision or $\lambda p\left(n_{t}, \Gamma\right)$ in the case of public funding. Equation (27) holds because at $t_{0}$ no sector can have a productivity parameter above $A_{t_{0}}^{\max }$ and equation (28) gives us the rate at which the sectors behind $A_{t_{0}}^{\max }$ innovate and get a productivity parameter larger than $A_{t_{0}}^{\max }$. These two equations define a differential equation whose solution is given by

$$
\Phi(t)=\exp \left(-\int_{t_{0}}^{t} d(s) d s\right) .
$$

We also know that $\frac{\dot{A}_{t}^{\max }}{A_{t}^{\max }}=\gamma(t)$, therefore

$$
A_{t}^{\max }=A_{t_{0}}^{\max } \exp \left(\int_{t_{0}}^{t} \gamma(s) d s\right) .
$$

Define $a_{0}=\frac{A_{t_{0}}^{\max }}{A_{t}^{\max }}$, then

$$
a_{0}=\exp \left(-\int_{t_{0}}^{t} \gamma(s) d s\right)
$$

Equation (30) defines an implicit function relating $t$ with $a_{0}$, the relative productivity parameter of a sector that innovated on date $t_{0}$. Let $t=\tilde{t}\left(a_{0}\right)$ be this function, and use it to perform a change of variable in (29) so that we will now have

$$
\Phi\left(\tilde{t}\left(a_{0}\right)\right)=\exp \left(-\int_{\tilde{t}^{-1}\left(t_{0}\right)}^{\tilde{t}^{-1}(t)} d\left(\tilde{t}\left(a_{0}\right)\right) \tilde{t}^{\prime}\left(a_{0}\right) d a_{0}\right) .
$$

Notice that this function is giving us the mass of sectors with a productivity parameter smaller or equal than $A_{t_{0}}^{\max }$ and that this is equivalent to the mass of sectors with a relative productivity parameter $a_{i t}$ below $a_{0}$. Therefore, we can redefine $\Phi\left(\tilde{t}\left(a_{0}\right)\right)=H\left(a_{0}\right)$ as the value of the distribution function for a sector that innovated on date $t_{0}$. After a long enough period of time, all sectors will have innovated at least once and therefore, $H(a)$ will be the distribution function of any sector with $a \in(0,1)$. Therefore, the long run distribution of relative productivity parameters across sectors will be given by

$$
H(a)=\exp \left(\int_{a}^{1} d(\tilde{t}(u)) \tilde{t}^{\prime}(u) d u\right)
$$


where we are using $\tilde{t}^{-1}(t)=a$ and $\tilde{t}^{-1}\left(t_{0}\right)=1$. Notice that this distribution is time invariant.

In general, we will not be able to obtain the functional form of $H(a)$ for any economic equilibrium. Nevertheless, in order to study the dynamics of the economy it is enough to know that the distribution is time invariant. However, we can get the expression of $H(a)$ when the economy is in a steady state, since in that case both the growth rate of the economy $\gamma$, and the probability of innovation $d$, are constant and thus (30) becomes

$$
a=\exp \left(-\gamma\left(t-t_{0}\right)\right)
$$

from where we can obtain the expression for $\tilde{t}(a)$ as given by

$$
t=-\frac{\ln a}{\gamma}+t_{0}
$$

which allows us to obtain the distribution function as

$$
H(a)=a^{\frac{d}{\gamma}}
$$

\section{B Proofs of propositions}

\section{B.1 Propositions under the public provision assumption}

Proof of Proposition 1. The effect on growth of $s_{n}$ is given by the following expression:

$$
\frac{d \gamma}{d s_{n}}=\frac{1}{2}\left(\frac{\beta \gamma}{n_{A}}\right)\left(1+\left(\frac{1-\beta}{\beta}\right) \frac{n_{A}}{n_{B}}\right) \frac{d n}{d s_{n}}
$$

Therefore, in order to find the sign of $\frac{d \gamma}{d s_{n}}$ we need first the sign of $\frac{d n}{d s_{n}}$. This derivative can be obtained from the system determining steady state equilibrium using implicit differentiation techniques. Consider the case of public provision of research. The relevant system of equations is the one formed by (21) and (22). Rewrite these equations in the following form:

$$
\begin{aligned}
f_{1}(k, n)=\left(1-s_{n}\right)\left[\gamma+\rho+\lambda p(n)+\lambda\left[\Gamma_{a}\left(1+b \Gamma_{b}\right)\right]^{\frac{1}{2}}\right]-\frac{\lambda p(n)(1-\alpha) \alpha L^{1-\alpha} k^{\alpha}}{n} & =0 \\
f_{2}(k, n)=\gamma+\rho+\delta+\tau_{k}-\alpha^{2} L^{1-\alpha} k^{\alpha-1} & =0,
\end{aligned}
$$

so that we may define the function $F:(0, \infty) \times(0, \infty) \rightarrow R^{2}$ whose components are $f_{1}(\cdot, \cdot)$ and $f_{2}(\cdot, \cdot)$ and use the implicit function theorem to find the derivatives needed. The Jacobian of $F$ will be given by

$$
J_{F}(k, n)=\left[\begin{array}{cc}
-\frac{\lambda p(n)(1-\alpha) \zeta}{n} & \left(1-s_{n}\right)\left(\lambda p^{\prime}(n)+\frac{d \gamma}{d n}\right)-\lambda \frac{d}{d n}\left(\frac{p(n)}{n}\right)(1-\alpha) \alpha L^{1-\alpha} k^{\alpha} \\
\frac{(1-\alpha) \zeta}{k} & \frac{d \gamma}{d n}
\end{array}\right],
$$


where $\frac{d \gamma}{d n}=\frac{1}{2}\left(\frac{\beta \gamma}{n_{A}}\right)\left(1+\left(\frac{1-\beta}{\beta}\right) \frac{n_{A}}{n_{B}}\right)$. The Jacobian may be inverted to obtain

$$
\left[J_{F}\right]^{-1}=\frac{1}{\operatorname{det}\left(J_{F}\right)}\left[\begin{array}{cc}
\frac{d \gamma}{d n} & -\left(1-s_{n}\right)\left(\lambda p^{\prime}(n)+\frac{d \gamma}{d n}\right)+\lambda \frac{d}{d n}\left(\frac{p(n)}{n}\right)(1-\alpha) \alpha L^{1-\alpha} k^{\alpha} \\
-\frac{(1-\alpha) \zeta}{k} & -\frac{\lambda p(n)(1-\alpha) \zeta}{n}
\end{array}\right],
$$

where

$$
\begin{array}{r}
\operatorname{det}\left(J_{F}\right)=-(1-\alpha) \zeta \frac{d \gamma}{d n}\left(\frac{\lambda p(n)}{n}+\frac{\left(1-s_{n}\right)}{k}\right) \\
-(1-\alpha) \zeta\left(\frac{\left(1-s_{n}\right) \lambda p^{\prime}(n)}{k}-\lambda \frac{d}{d n}\left(\frac{p(n)}{n}\right) \alpha(1-\alpha) L^{1-\alpha} k^{\alpha-1}\right),
\end{array}
$$

is negative. The derivatives of $F$ with respect to $s_{n}$ are given by

$$
\begin{array}{r}
\frac{d f_{1}}{d s_{n}}=-\left[\gamma+\rho+\lambda p(n)+\lambda\left[\Gamma_{a}\left(1+b \Gamma_{b}\right)\right]^{\frac{1}{2}}\right] \\
\frac{d f_{2}}{d s_{n}}=0 .
\end{array}
$$

Therefore, $\frac{d n}{d s_{n}}$ will be given by

$$
\frac{d n}{d s_{n}}=\frac{-1}{\operatorname{det}\left(J_{F}\right)}\left(\frac{(1-\alpha) \zeta}{k}\right)\left[\gamma+\rho+\lambda p(n)+\lambda\left[\Gamma_{a}\left(1+b \Gamma_{b}\right)\right]^{\frac{1}{2}}\right],
$$

which is positive. Therefore, the derivative of the steady state rate of growth with respect to $s_{n}$ is also positive.

Proof of Proposition 2. The effect on growth of public basic research is given by

$$
\frac{d \gamma}{d \Gamma_{b}}=\left(\frac{(1-\beta) \gamma}{n_{B}}\right)\left(1+\frac{1}{2}\left(1+\left(\frac{\beta}{1-\beta}\right) \frac{n_{B}}{n_{A}}\right) \frac{d n}{d \Gamma_{b}}\right) .
$$

Accordingly, let us find $\frac{d n}{d \Gamma_{b}}$. The Jacobian of $F$ is not modified but we have to compute the derivatives of $F$ with respect to $\Gamma_{b}$. They are given by the following expressions:

$$
\begin{array}{r}
\frac{d f_{1}}{d \Gamma_{b}}=\left(1-s_{n}\right)\left[\frac{(1-\beta) \gamma}{n_{B}}+\frac{\lambda b^{\frac{1}{2}}}{2}\left(\frac{b \Gamma_{a}}{1+b \Gamma_{b}}\right)^{\frac{1}{2}}\right] \\
\frac{d f_{2}}{d \Gamma_{b}}=\frac{(1-\beta) \gamma}{n_{B}},
\end{array}
$$

which implies that the derivative of private research with respect to public basic research, as expressed by

$$
\frac{d n}{d \Gamma_{b}}=\frac{(1-\alpha) \zeta\left[\left(\frac{1-s_{n}}{k}\right)\left(\frac{\lambda b^{\frac{1}{2}}}{2}\left(\frac{b \Gamma_{a}}{1+b \Gamma_{b}}\right)^{\frac{1}{2}}\right)+\left(\frac{\lambda p(n)}{n}+\frac{1-s_{n}}{k}\right)\left(\frac{(1-\beta) \gamma}{n_{B}}\right)\right]}{\operatorname{det}\left(J_{F}\right)},
$$

is negative. The derivative of $\gamma$ with respect to $\Gamma_{b}$ is therefore,

$$
\frac{d \gamma}{d \Gamma_{b}}=\frac{\left(\frac{(1-\beta) \gamma}{n_{B}}\right)\left(\left(\frac{1-s_{n}}{k}\right)\left(\frac{\lambda b^{\frac{1}{2}}}{2}\right)\left(1-\frac{1}{2}\left(1+\left(\frac{\beta}{1-\beta}\right) \frac{n_{B}}{n_{A}}\right)\left(\frac{b \Gamma_{a}}{1+b \Gamma_{b}}\right)^{\frac{1}{2}}\right)+\frac{\lambda \alpha(1-\alpha) L^{1-\alpha} k^{\alpha-1}}{2 \sqrt{b} n^{2}}\right)}{\frac{d \gamma}{d n}\left(\frac{\lambda p(n)}{n}+\frac{\left(1-s_{n}\right)}{k}\right)+\frac{\left(1-s_{n}\right)}{k}\left(\frac{\lambda b^{\frac{1}{2}}}{2}\right)+\frac{\lambda \alpha(1-\alpha) L^{1-\alpha} k^{\alpha-1}}{2 \sqrt{b} n^{2}}} .
$$


If public applied research is zero, $\frac{d \gamma}{d \Gamma_{b}}$ is positive. However, if $\Gamma_{a}$ is positive, the effect on growth of public basic research will be positive whenever

$$
\left(\frac{1-s_{n}}{k}\right)\left(\frac{\lambda b^{\frac{1}{2}}}{2}\right)\left(1-\frac{1}{2}\left(1+\left(\frac{\beta}{1-\beta}\right) \frac{n_{B}}{n_{A}}\right)\left(\frac{b \Gamma_{a}}{1+b \Gamma_{b}}\right)^{\frac{1}{2}}\right)+\frac{\lambda \alpha(1-\alpha) L^{1-\alpha} k^{\alpha-1}}{2 \sqrt{b} n^{2}}>0 .
$$

A sufficient condition for $\frac{d \gamma}{d \Gamma_{b}}>0$ would be

$$
\frac{1}{2}\left(1+\left(\frac{\beta}{1-\beta}\right) \frac{n_{B}}{n_{A}}\right)\left(\frac{b \Gamma_{a}}{1+b \Gamma_{b}}\right)^{\frac{1}{2}} \leq 1
$$

but this expression still depends upon $n$. Recall that $\frac{n_{B}}{n_{A}}=\frac{b n-1+2 b \Gamma_{b}}{1+b n+2 b \Gamma_{a}}$. For given values of public basic and applied research, $\frac{n_{B}}{n_{A}}$ is a function of $n$ whose derivative is given by $\frac{d}{d n}\left(\frac{n_{B}}{n_{A}}\right)=\frac{2 b\left(1+b\left(\Gamma_{a}-\Gamma_{b}\right)\right)}{\left(1+b n+2 b \Gamma_{a}\right)^{2}}$. Therefore, $\frac{n_{B}}{n_{A}}$ is an increasing function of $n$ when $1+b\left(\Gamma_{a}-\Gamma_{b}\right)>0$ and a decreasing function when $1+b\left(\Gamma_{a}-\Gamma_{b}\right)<0$. Consider $\frac{n_{B}}{n_{A}}$ increasing. Then it will take its maximum value when $n$ goes to infinity. Since $\lim _{n \rightarrow \infty} \frac{n_{B}}{n_{A}}=1$, a sufficient condition for (35) to be satisfied is

$$
\frac{1}{2(1-\beta)}\left(\frac{b \Gamma_{a}}{1+b \Gamma_{b}}\right)^{\frac{1}{2}} \leq 1
$$

Consider now the case when $\frac{n_{B}}{n_{A}}$ is decreasing in $n$, that is when $1+b\left(\Gamma_{a}-\Gamma_{b}\right)<0$. In this case, $\frac{n_{B}}{n_{A}}$ will take its maximum value at $n=\frac{1}{b}$ (for the range of values that we are considering) and condition (35) will be satisfied if

$$
\frac{1}{2}\left(1+\left(\frac{\beta}{1-\beta}\right) \frac{b \Gamma_{b}}{1+b \Gamma_{a}}\right)\left(\frac{b \Gamma_{a}}{1+b \Gamma_{b}}\right)^{\frac{1}{2}} \leq 1
$$

It follows that if $\psi \in \Psi_{1}$, condition (34) is satisfied and $\frac{d \gamma}{d \Gamma_{b}}>0$.

In order to prove the third part of the proposition, we have to find a sufficient condition for $\frac{d \gamma}{d \Gamma_{b}}<0$. Notice that in equilibrium $f_{1}(k, n)=0$ and therefore

$$
\frac{\lambda \alpha(1-\alpha) L^{1-\alpha} k^{\alpha-1}}{2 \sqrt{b} n^{2}}=\left(\frac{1-s_{n}}{k}\right)\left(\frac{\rho+\gamma+\lambda p(n)+\lambda\left(\Gamma_{a}\left(1+b \Gamma_{b}\right)\right)^{\frac{1}{2}}}{n(1+b n)}\right),
$$

which recalling (34) allows us to state that $\frac{d \gamma}{d \Gamma_{b}}$ will be negative whenever

$$
\left[\frac{1}{2}\left(\frac{b \Gamma_{a}}{1+b \Gamma_{b}}\right)^{\frac{1}{2}}\right]>1+\frac{\gamma+\rho+\lambda p(n)+\lambda\left[\Gamma_{a}\left(1+b \Gamma_{b}\right)\right]^{\frac{1}{2}}}{\lambda p(n) b n} .
$$

Notice that the numerator of the last expression of (36) is the discount rate of the flow of profits, which for reasonable values of the parameters should be smaller than 1 . In order to impose this condition, define $n^{1}$ as the level of research intensity implying a discount rate of 1 . Then, a sufficient condition for the discount rate to be smaller than 1 is $n<n^{1}$ or, equivalently, ${ }^{13}$

$$
\chi_{1}>\chi_{2},
$$

\footnotetext{
${ }^{13}$ This condition is obtained from the equations that determine the equilibrium value of $n$, that is from (31) and (32).
} 
where

$$
\chi_{1}=\left(\frac{\left(1-s_{n}\right)\left(\gamma\left(n^{1}\right)+\rho+\lambda p\left(n^{1}\right)+\lambda\left(\Gamma_{a}\left(1+b \Gamma_{b}\right)\right)^{\frac{1}{2}}\right)}{\lambda b p\left(n^{1}\right)(1-\alpha) \alpha L^{1-\alpha}}\right)^{\frac{1}{\alpha}}
$$

and

$$
\chi_{2}=\left(\frac{\alpha^{2} L^{1-\alpha}}{\gamma\left(n^{1}\right)+\rho+\delta+\tau_{k}}\right)^{\frac{1}{1-\alpha}}
$$

After having imposed this upper bound for $n$, a sufficient condition for (36) to hold is

$$
\left[\frac{1}{2}\left(\frac{b \Gamma_{a}}{1+b \Gamma_{b}}\right)^{\frac{1}{2}}\right]>1+\frac{1}{\lambda p(n) b n} .
$$

This expression implies that if $\Gamma_{a}$ is large relative to $\Gamma_{b}$, the effect on growth of public basic research will be negative as long as the level of private research intensity is not so small that $\frac{1}{\lambda p(n) b n}$ becomes excessively large. Therefore, what we are requiring is that $\Gamma_{a}$ is large relative to $\Gamma_{b}$ but also that they both are not too large. If we want to find a sufficient condition that depends only on the values of the parameters we have to impose a lower bound for $n$. Let $n^{0}$ be the level of research intensity that satisfies $\frac{1}{\lambda p(n) b n}=\epsilon$, where $\epsilon$ is a real number. ${ }^{14}$ Then, if $n>n^{0}$, condition (36) will be satisfied when

$$
\left[\frac{1}{2}\left(\frac{b \Gamma_{a}}{1+b \Gamma_{b}}\right)^{\frac{1}{2}}\right]>1+\epsilon
$$

In addition, we have to impose the following restriction on the parameters in order to guarantee $n>n^{0}$ :

$$
\chi_{3}<\chi_{4}
$$

where

$$
\chi_{3}=\left(\frac{\left(1-s_{n}\right)\left(\gamma\left(n^{0}\right)+\rho+\lambda p\left(n^{0}\right)+\lambda\left(\Gamma_{a}\left(1+b \Gamma_{b}\right)\right)^{\frac{1}{2}}\right)}{\lambda b p\left(n^{0}\right)(1-\alpha) \alpha L^{1-\alpha}}\right)^{\frac{1}{\alpha}}
$$

and

$$
\chi_{4}=\left(\frac{\alpha^{2} L^{1-\alpha}}{\gamma\left(n^{0}\right)+\rho+\delta+\tau_{k}}\right)^{\frac{1}{1-\alpha}}
$$

Thus, if $\omega \in \Omega_{1}$ then $\frac{d \gamma}{d \Gamma_{b}}<0$.

Proof of Proposition 3. The derivative of the growth rate with respect to public applied research is given by

$$
\frac{d \gamma}{d \Gamma_{a}}=\left(\frac{\beta \gamma}{n_{A}}\right)\left(1+\frac{1}{2}\left(1+\left(\frac{1-\beta}{\beta}\right) \frac{n_{A}}{n_{B}}\right) \frac{d n}{d \Gamma_{a}}\right) .
$$

\footnotetext{
${ }^{14}$ The choice of $\epsilon$ must take into account that if it is either too large or too small the set of parameter values satisfying the condition may be empty. For a standard set of parameter values $\epsilon=1$, for instance, yields a non-empty set.
} 
As in the previous propositions we compute first the derivative of private research intensity with respect to public applied research. In order to do so we need the derivatives of the component functions of $F$, i.e.

$$
\begin{array}{r}
\frac{d f_{1}}{d \Gamma_{a}}=\left(1-s_{n}\right)\left[\frac{\beta \gamma}{n_{A}}+\left(\frac{\lambda b^{\frac{1}{2}}}{2}\right)\left(\frac{1+b \Gamma_{b}}{b \Gamma_{a}}\right)^{\frac{1}{2}}\right] \\
\frac{d f_{2}}{d \Gamma_{a}}=\frac{\beta \gamma}{n_{A}} .
\end{array}
$$

Next, pre-multiply $\left(\begin{array}{c}\frac{d f_{1}}{d \Gamma_{a}} \\ \frac{d f_{2}}{d \Gamma_{a}}\end{array}\right)$ by the second row of $-\left[J_{F}\right]^{-1}$ to obtain

$$
\frac{d n}{d \Gamma_{a}}=\left(\frac{(1-\alpha) \zeta}{\operatorname{det}\left(J_{F}\right)}\right)\left[\left(\frac{\left(1-s_{n}\right)}{k}\right)\left(\frac{\lambda b^{\frac{1}{2}}}{2}\right)\left(\frac{1+b \Gamma_{b}}{b \Gamma_{a}}\right)^{\frac{1}{2}}+\left(\frac{\lambda p(n)}{n}+\frac{\left(1-s_{n}\right)}{k}\right)\left(\frac{\beta \gamma}{n_{A}}\right)\right] .
$$

Notice that this expression is also negative. Now we can write $\frac{d \gamma}{d \Gamma_{a}}$ as follows:

$$
\frac{d \gamma}{d \Gamma_{a}}=\frac{\left(\frac{\beta \gamma}{n_{A}}\right)\left(\left(\frac{1-s_{n}}{k}\right)\left(\frac{\lambda b^{\frac{1}{2}}}{2}\right)\left(1-\frac{1}{2}\left(1+\left(\frac{1-\beta}{\beta}\right) \frac{n_{A}}{n_{B}}\right)\left(\frac{1+b \Gamma_{b}}{b \Gamma_{a}}\right)^{\frac{1}{2}}\right)+\frac{\lambda \alpha(1-\alpha) L^{1-\alpha} k^{\alpha-1}}{2 \sqrt{b} n^{2}}\right)}{\frac{d \gamma}{d n}\left(\frac{\lambda p(n)}{n}+\frac{\left(1-s_{n}\right)}{k}\right)+\frac{\left(1-s_{n}\right)}{k}\left(\frac{\lambda b^{\frac{1}{2}}}{2}\right)+\frac{\lambda \alpha(1-\alpha) L^{1-\alpha} k^{\alpha-1}}{2 n^{2} \sqrt{b}}} .
$$

Therefore, public applied research will have a positive effect on growth only if

$$
\left(\frac{1-s_{n}}{k}\right)\left(\frac{\lambda b^{\frac{1}{2}}}{2}\right)\left(1-\frac{1}{2}\left(1+\left(\frac{1-\beta}{\beta}\right) \frac{n_{A}}{n_{B}}\right)\left(\frac{1+b \Gamma_{b}}{b \Gamma_{a}}\right)^{\frac{1}{2}}\right)+\frac{\lambda \alpha(1-\alpha) L^{1-\alpha} k^{\alpha-1}}{2 n^{2} \sqrt{b}}
$$

is positive.

A sufficient condition for the expression in (41) to be positive is

$$
1-\frac{1}{2}\left(1+\left(\frac{1-\beta}{\beta}\right) \frac{n_{A}}{n_{B}}\right)\left(\frac{1+b \Gamma_{b}}{b \Gamma_{a}}\right)^{\frac{1}{2}}>0 .
$$

Given that $\frac{n_{A}}{n_{B}}$ as a function of $n$ is increasing when $1+b\left(\Gamma_{a}-\Gamma_{b}\right)<0$ and decreasing when $1+b\left(\Gamma_{a}-\Gamma_{b}\right)>$ 0 , it will take its maximum value when $n$ goes to infinity in the first case and when $n=\frac{1}{b}$ in the second case. Therefore, sufficient conditions for (42) to be satisfied are

$$
\begin{aligned}
& \frac{1}{2 \beta}\left(\frac{1+b \Gamma_{b}}{b \Gamma_{a}}\right)^{\frac{1}{2}}<1 \quad \text { for } 1+b\left(\Gamma_{a}-\Gamma_{b}\right)<0 \\
& \frac{1}{2}\left(\frac{1+b \Gamma_{b}}{b \Gamma_{a}}\right)^{\frac{1}{2}}\left(1+\left(\frac{1-\beta}{\beta}\right) \frac{1+b \Gamma_{a}}{b \Gamma_{b}}\right)<1 \quad \text { for } \quad 1+b\left(\Gamma_{a}-\Gamma_{b}\right)>0
\end{aligned}
$$

Under these conditions, $\frac{d \gamma}{d \Gamma_{a}}>0$. Thus, if $\psi \in \Psi_{2}$, the growth effect of public applied research will be positive.

The expression of $\frac{d \gamma}{d \Gamma_{a}}$ in equation (40) implies that this derivative will be negative whenever

$$
\left(\frac{1-s_{n}}{k}\right)\left(\frac{\lambda b^{\frac{1}{2}}}{2}\right)\left(1-\frac{1}{2}\left(1+\left(\frac{1-\beta}{\beta}\right) \frac{n_{A}}{n_{B}}\right)\left(\frac{1+b \Gamma_{b}}{b \Gamma_{a}}\right)^{\frac{1}{2}}\right)+\frac{\lambda \alpha(1-\alpha) L^{1-\alpha} k^{\alpha-1}}{2 \sqrt{b} n^{2}}<0 .
$$


Therefore, following the same reasoning as in the previous proofs, the effect on growth of public applied research will be negative when $\omega \in \Omega_{2}$.

Proof of Proposition 4. The derivatives of $f_{1}(k, n)$ and $f_{2}(k, n)$ with respect to $\Gamma$ are given by the following expressions:

$$
\begin{array}{r}
\frac{d f_{1}}{d \Gamma}=\left(1-s_{n}\right)\left(\frac{d \gamma}{d n}+\lambda p^{\prime}(\Gamma)\right) \\
\frac{d f_{2}}{d \Gamma}=\frac{d \gamma}{d n},
\end{array}
$$

where we are using the fact that under these assumptions, the derivative of the growth rate with respect to $\Gamma$ keeping $n$ constant is equal to the derivative of the growth rate with respect to $n$. Given (44) and (45), the derivative of private research with respect to public research is

$$
\frac{d n}{d \Gamma}=\frac{(1-\alpha) \zeta}{\operatorname{det}\left(J_{F}\right)}\left(\frac{d \gamma}{d n}\left(\frac{1-s_{n}}{k}+\frac{\lambda p(n)}{n}\right)+\frac{1-s_{n}}{k} \lambda p^{\prime}(\Gamma)\right),
$$

which is negative. Given $\frac{d n}{d \Gamma}$, we can express the growth derivative as

$$
\frac{d \gamma}{d \Gamma}=\frac{d \gamma}{d n}\left(1+\frac{d n}{d \Gamma}\right)=\frac{d \gamma}{d n}\left(-\frac{(1-\alpha) \zeta}{\operatorname{det}\left(J_{F}\right)}\right)\left(\frac{-\lambda \tilde{\pi}}{k} \frac{d}{d n}\left(\frac{p(n)}{n}\right)\right),
$$

where $\tilde{\pi}=\frac{\pi}{A_{t}^{\max }}$. Since $\frac{d}{d n}\left(\frac{p(n)}{n}\right)$ and $\operatorname{det}\left(J_{F}\right)$ are negative, then $\frac{d \gamma}{d \Gamma}>0$.

\section{B.2 Propositions under the public funding assumption}

Proof of Proposition 5. The relevant equations under this assumption are (21) and (23) so that the component functions of $F$ are now

$$
\begin{aligned}
& f_{1}(k, n)=\left(1-s_{n}\right)[\gamma+\rho+\lambda p(n, \Gamma)]-\frac{\lambda p(n, \Gamma)}{n}(1-\alpha) \alpha L^{1-\alpha} k^{\alpha}=0 \\
& f_{2}(k, n)=\gamma+\rho+\delta+\tau_{k}-\alpha^{2} L^{1-\alpha} k^{\alpha-1}=0 .
\end{aligned}
$$

Hence, the Jacobian and its inverse are given by the following matrices:

$$
J_{F}(k, n)=\left[\begin{array}{cc}
-\frac{\lambda p(n, \Gamma)(1-\alpha) \zeta}{n} & \left(1-s_{n}\right)\left(\lambda p^{\prime}(n, \Gamma)+\frac{d \gamma}{d n}\right)-\lambda \frac{d}{d n}\left(\frac{p(n, \Gamma)}{n}\right)(1-\alpha) \alpha L^{1-\alpha} k^{\alpha} \\
\frac{(1-\alpha) \zeta}{k} & \frac{d \gamma}{d n}
\end{array}\right]
$$

and

$$
\left[J_{F}\right]^{-1}=\frac{1}{\operatorname{det}\left(J_{F}\right)}\left[\begin{array}{cc}
\frac{d \gamma}{d n} & -\left(1-s_{n}\right)\left(\lambda p^{\prime}(n, \Gamma)+\frac{d \gamma}{d n}\right)+\lambda \frac{d}{d n}\left(\frac{p(n, \Gamma)}{n}\right)(1-\alpha) \alpha L^{1-\alpha} k^{\alpha} \\
-\frac{(1-\alpha) \zeta}{k} & -\frac{\lambda p(n, \Gamma)(1-\alpha) \zeta}{n}
\end{array}\right]
$$


where

$$
\begin{aligned}
\operatorname{det}\left(J_{F}\right)=-(1-\alpha) \zeta \frac{d \gamma}{d n}\left[\frac{\left(1-s_{n}\right)}{k}\right. & \left.+\frac{\lambda p(n, \Gamma)}{n}\right]- \\
& -(1-\alpha) \zeta\left(\frac{\left(1-s_{n}\right) \lambda p^{\prime}(n, \Gamma)}{k}-\lambda \frac{d}{d n}\left(\frac{p(n, \Gamma)}{n}\right)(1-\alpha) \alpha L^{1-\alpha} k^{\alpha-1}\right)
\end{aligned}
$$

is also negative.

The derivatives of the component functions of $F$ with respect to $s_{n}$ are

$$
\begin{aligned}
\frac{d f_{1}}{d s_{n}} & =-[\gamma+\rho+\lambda p(n, \Gamma)] \\
\frac{d f_{2}}{d s_{n}} & =0 .
\end{aligned}
$$

Thus, $\frac{d n}{d s_{n}}$ is given by

$$
\frac{d n}{d s_{n}}=-\left(\frac{(1-\alpha) \zeta}{\operatorname{det}\left(J_{F}\right)}\right)\left(\frac{\gamma+\rho+\lambda p(n, \Gamma)}{k}\right) .
$$

Therefore, private research intensity increases with subsidies to research and so does the growth rate of the economy.

Proof of Proposition 6. Recall that $\Gamma=\Gamma_{a}+\Gamma_{b}$. Given that both applied and basic public research enter the component functions in equivalent positions, the derivatives of private research intensity with respect to $\Gamma_{a}$ and $\Gamma_{b}$ will be identical. In addition, the equilibrium expression for the growth rate under this assumption is given by

$$
\gamma=\sigma \lambda\left(\frac{1+b\left(n+\Gamma_{a}+\Gamma_{b}\right)}{2 b}\right)^{\beta}\left(\frac{b\left(n+\Gamma_{a}+\Gamma_{b}\right)-1}{2 b}\right)^{1-\beta} .
$$

Consequently,

$$
\frac{d \gamma}{d \Gamma_{a}}=\frac{d \gamma}{d \Gamma_{b}}=\frac{d \gamma}{d \Gamma}=\frac{d \gamma}{d n}\left(1+\frac{d n}{d \Gamma}\right)
$$

Therefore, we can talk about $\Gamma$ exclusively.

In order to obtain $\frac{d n}{d \Gamma}$ we compute the derivatives of the component functions of $F$ as follows:

$$
\begin{aligned}
& \frac{d f_{1}}{d \Gamma}=\left(1-s_{n}\right)\left[\frac{d \gamma}{d n}+\lambda p^{\prime}(n, \Gamma)\right]-\frac{\lambda p^{\prime}(n, \Gamma) \tilde{\pi}}{n} \\
& \frac{d f_{2}}{d \Gamma}=\frac{d \gamma}{d n}
\end{aligned}
$$

where $\tilde{\pi}=\frac{\pi}{A_{t}^{\max }}$. Therefore, the derivative of private research with respect to public research is given by

$$
\frac{d n}{d \Gamma}=\frac{-\frac{d \gamma}{d n}\left(\frac{\left(1-s_{n}\right)}{k}+\frac{\lambda p(n, \Gamma)}{n}\right)+\frac{\lambda p^{\prime}(n, \Gamma) \tilde{\pi}}{n k}-\frac{\left(1-s_{n}\right) \lambda p^{\prime}(n, \Gamma)}{k}}{\frac{d \gamma}{d n}\left[\frac{\left(1-s_{n}\right)}{k}+\frac{\lambda p(n, \Gamma)}{n}\right]+\frac{\left(1-s_{n}\right) \lambda p^{\prime}(n, \Gamma)}{k}-\lambda \frac{d}{d n}\left(\frac{p(n, \Gamma)}{n}\right) \frac{\tilde{\pi}}{k}} .
$$

Notice that the sign of this derivative is ambiguous, which implies that whether public research crowds out private research or not, depends on the values of the parameters and the initial situation of the economy. 
Given $\frac{d n}{d \Gamma}$, it is immediate from (46) that the derivative of the growth rate with respect to public funding of research is positive and equal to

$$
\frac{d \gamma}{d \Gamma}=\frac{\left(\frac{\lambda \tilde{\pi}}{k}\right) \frac{d \gamma}{d n}\left(\frac{p^{\prime}(n, \Gamma)}{n}-\frac{d}{d n}\left(\frac{p(n, \Gamma)}{n}\right)\right)}{\frac{d \gamma}{d n}\left[\frac{\left(1-s_{n}\right)}{k}+\frac{\lambda p(n, \Gamma)}{n}\right]+\frac{\left(1-s_{n}\right) \lambda p^{\prime}(n, \Gamma)}{k}-\lambda \frac{d}{d n}\left(\frac{p(n, \Gamma)}{n}\right) \frac{\tilde{\pi}}{k}} .
$$

Proof of Proposition 7. In order to compare the growth effects of $s_{n}$ and $\Gamma$, they must have an equivalent impact on the public budget. So let us consider as the initial situation the equilibrium corresponding to $s_{n}=0$ and $\Gamma=0$. In this situation, we must compare $\frac{d \gamma}{d \Gamma}$ and $\frac{d \gamma}{d s_{n}}\left(\frac{1}{n}\right)$. Recall that

$$
\begin{aligned}
\frac{d \gamma}{d s_{n}} & =-\frac{d \gamma}{d n}\left(\frac{(1-\alpha) \zeta}{\operatorname{det}\left(J_{F}\right)}\right)\left(\frac{\gamma+\rho+\lambda p(n, \Gamma)}{k}\right) \\
\frac{d \gamma}{d \Gamma} & =\frac{d \gamma}{d n}\left(\frac{(1-\alpha) \zeta}{\operatorname{det}\left(J_{F}\right)}\right)\left(\lambda \frac{d}{d n}\left(\frac{p(n, \Gamma)}{n}\right) \frac{\tilde{\pi}}{k}-\frac{\lambda p^{\prime}(n, \Gamma) \tilde{\pi}}{n k}\right) .
\end{aligned}
$$

Notice also that from $f_{1}(k, n)=0, \lambda \frac{\tilde{\pi}}{k}\left(\frac{p^{\prime}(n, \Gamma)}{n}-\frac{d}{d n}\left(\frac{p(n, \Gamma)}{n}\right)\right)=\left(1-s_{n}\right)\left(\frac{\gamma+\rho+\lambda p(n, \Gamma)}{n k}\right)$, therefore

$$
\frac{d \gamma}{d \Gamma}=-\frac{d \gamma}{d n}\left(\frac{(1-\alpha) \zeta}{\operatorname{det}\left(J_{F}\right)}\right)\left(1-s_{n}\right)\left(\frac{\gamma+\rho+\lambda p(n, \Gamma)}{n k}\right)
$$

Since we are considering $s_{n}=0, \frac{d \gamma}{d s_{n}}\left(\frac{1}{n}\right)=\frac{d \gamma}{d \Gamma}$.

Proof of Corollary 8. Recall that

$$
\begin{aligned}
\frac{d \gamma}{d s_{n}} & =\frac{d \gamma}{d n}\left(\frac{d n}{d s_{n}}\right) \\
\frac{d \gamma}{d \Gamma} & =\frac{d \gamma}{d n}\left(1+\frac{d n}{d \Gamma}\right)
\end{aligned}
$$

Therefore, if $\frac{d \gamma}{d s_{n}}\left(\frac{1}{n}\right)=\frac{d \gamma}{d \Gamma}$, then $\frac{d n}{d s_{n}}\left(\frac{1}{n}\right)=1+\frac{d n}{d \Gamma}$ and $\frac{d n}{d \Gamma}=\frac{d n}{d s_{n}}\left(\frac{1}{n}\right)-1$.

\section{Calibration}

\section{C.1 Public provision of research}

Tables 1 to 7 show the results of the calibration of the model for the following set of parameters:

- Capital intensity, $\alpha=0.7$. Therefore, we are considering a broad concept of capital that could include human capital.

- Contribution of applied research to technological change, $\beta=0.55$.

- The discount rate and the depreciation rate are the standard values of $\rho=0.02$ and $\delta=0.05$. 
- Other parameter values are: $\lambda=0.05, b=5, \sigma=\ln (1.2), \tau_{k}=0, L=1$. They were chosen so that the rate of growth of the economy, the interest rate and the level of consumption were positive and in a reasonable range.

- Regarding the choice of the range of values for public research, we took as reference the value of private research intensity when $s_{n}=\Gamma_{a}=\Gamma_{b}=0$. At this equilibrium, $n=7.143$.

- The following tables and figures present the welfare effects of the different policy instruments. By default, the policy instruments that are not being analyzed are set to zero, except when indicated.

Table 1. Welfare effect of the research subsidy

$\begin{array}{ll}\text { 1a) } \lambda=0.05 & \text { 1b) } \lambda=0.1\end{array}$

\begin{tabular}{|c|c|c|c|c|c|c|c|}
\hline$s_{n}$ & $\frac{d c}{d s_{n}}$ & $\frac{d V_{s}}{d s_{n}}$ & $\frac{d V}{d s_{n}}$ & $s_{n}$ & $\frac{d c}{d s_{n}}$ & $\frac{d V_{s}}{d s_{n}}$ & $\frac{d V}{d s_{n}}$ \\
\hline 0.0 & -15.35 & -1.53 & 13.77 & 0.0 & -11.55 & 8.81 & 27.08 \\
\hline 0.1 & -16.93 & -5.34 & 12.17 & 0.1 & -12.58 & 6.34 & 27.11 \\
\hline 0.2 & -18.90 & -12.5 & 7.88 & 0.2 & -13.83 & 0.77 & 24.73 \\
\hline 0.3 & -21.34 & -26.8 & -2.76 & 0.3 & -15.41 & -12.00 & 16.14 \\
\hline 0.4 & -24.59 & -59.8 & -30.59 & 0.4 & -17.50 & -44.30 & -10.4 \\
\hline 0.5 & -29.1 & -156.9 & -120.2 & 0.5 & -20.30 & -148.6 & -106.4 \\
\hline 0.6 & -35.7 & -748.2 & -699.8 & 0.6 & -24.50 & -920.0 & -864.7 \\
\hline
\end{tabular}

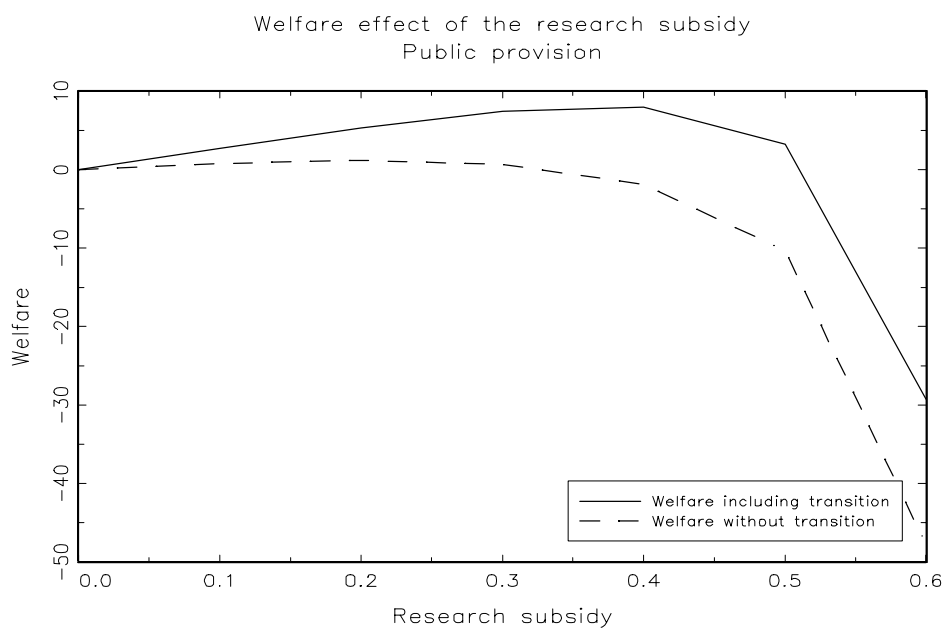

Figure 1: Welfare effect of the research subsidy under public provision. $(\lambda=0.1)$ 
Table 2. Welfare effect of basic research when $\Gamma_{a}=0$

\begin{tabular}{|c|c|c|c|c|}
\hline$\Gamma_{b}$ & $\frac{d c}{d \Gamma_{b}}$ & $\frac{d V_{s}}{d \Gamma_{b}}$ & $\frac{d V}{d \Gamma_{b}}$ & $n$ \\
\hline 0 & -1.92 & -0.65 & 1.10 & 7.14 \\
0.5 & -1.85 & -1.15 & 0.51 & 6.93 \\
1 & -1.79 & -1.65 & -0.08 & 6.74 \\
1.5 & -1.75 & -2.18 & -0.68 & 6.56 \\
2 & -1.70 & -2.74 & -1.29 & 6.40 \\
2.5 & -1.67 & -3.34 & -1.94 & 6.25 \\
3 & -1.64 & -3.99 & -2.63 & 6.11 \\
4 & -1.59 & -5.54 & -4.23 & 5.85 \\
6 & -1.52 & -10.66 & -9.29 & 5.43 \\
\hline
\end{tabular}

Welfare effect of public basic research

Public provision. Public applied research equal to 0

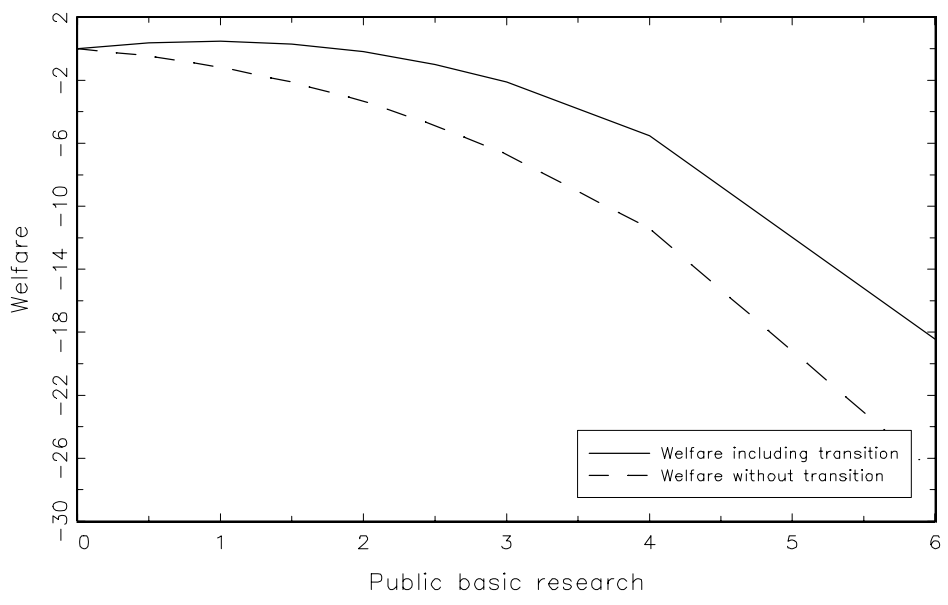

Figure 2: Welfare effect of public basic research $\left(\Gamma_{a}=0\right)$. 
Table 3. Welfare effect of basic research when $\Gamma_{a}=0.5$

\begin{tabular}{|c|c|c|c|c|c|}
\hline$\Gamma_{b}$ & $\frac{d c}{d \Gamma_{b}}$ & $\frac{d V_{s}}{d \Gamma_{b}}$ & $\frac{d V}{d \Gamma_{b}}$ & $\gamma$ & $n$ \\
\hline 0 & 1.05 & 0.20 & -0.78 & 0.03241 & 6.57 \\
0.1 & 0.50 & 0.05 & -0.44 & 0.03232 & 6.46 \\
0.2 & 0.20 & -0.05 & -0.25 & 0.03228 & 6.35 \\
0.5 & -0.31 & -0.28 & -0.05 & 0.03230 & 6.07 \\
1 & -0.64 & -0.59 & -0.12 & 0.03253 & 5.67 \\
1.5 & -0.78 & -0.88 & -0.34 & 0.03285 & 5.33 \\
2 & -0.86 & -1.16 & -0.60 & 0.03320 & 5.02 \\
2.5 & -0.90 & -1.44 & -0.89 & 0.03354 & 4.75 \\
3 & -0.92 & -1.72 & -1.19 & 0.03387 & 4.49 \\
\hline
\end{tabular}

Relationship between welfare and public basic research

Public Provision. Public applied research positive

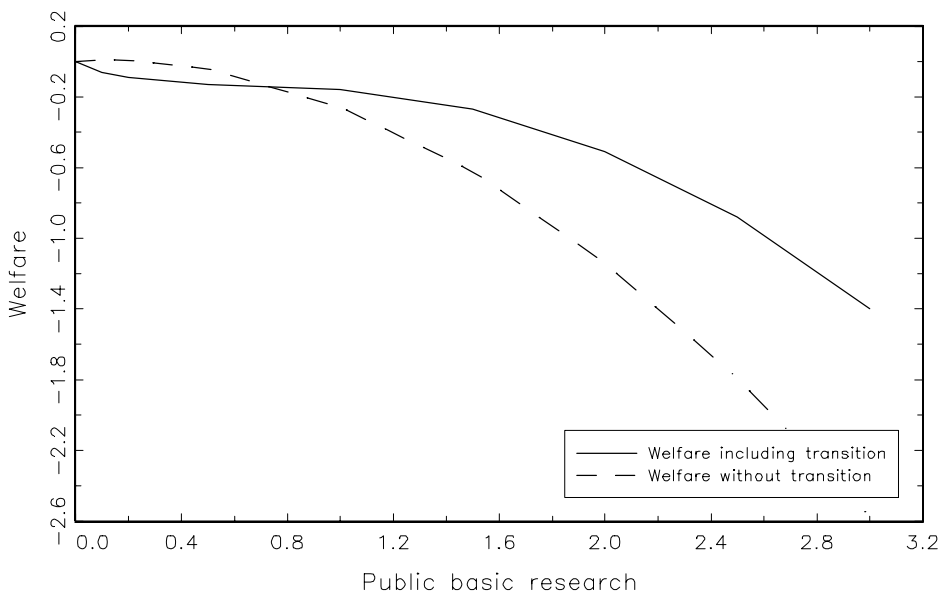

Figure 3: Welfare effect of public basic research $\left(\Gamma_{a}=0.5\right)$. 
Table 4. Welfare effect of applied research.

\begin{tabular}{|c|c|c|c|c|c|}
\hline$\Gamma_{a}$ & $\frac{d c}{d \Gamma_{a}}$ & $\frac{d V_{s}}{d \Gamma_{a}}$ & $\frac{d V}{d \Gamma_{a}}$ & $\gamma$ & $n$ \\
\hline 0 & -- & -- & -- & 0.03264 & 7.14 \\
$10^{-8}$ & 8628 & 1940 & -6314 & 0.03264 & 7.14 \\
0.01 & 6.58 & 1.58 & -4.63 & 0.03246 & 7.09 \\
0.1 & 0.68 & 0.31 & -0.27 & 0.03223 & 6.95 \\
0.2 & -0.10 & 0.13 & 0.27 & 0.03221 & 6.84 \\
0.5 & -0.78 & -0.14 & 0.61 & 0.03241 & 6.58 \\
1 & -1.75 & -0.48 & 0.49 & 0.03297 & 6.21 \\
2 & -1.20 & -1.17 & -0.15 & 0.03424 & 5.61 \\
4 & -1.23 & -2.66 & -1.76 & 0.03658 & 4.69 \\
10 & -1.14 & -11.50 & -10.85 & 0.04105 & 3.03 \\
\hline
\end{tabular}

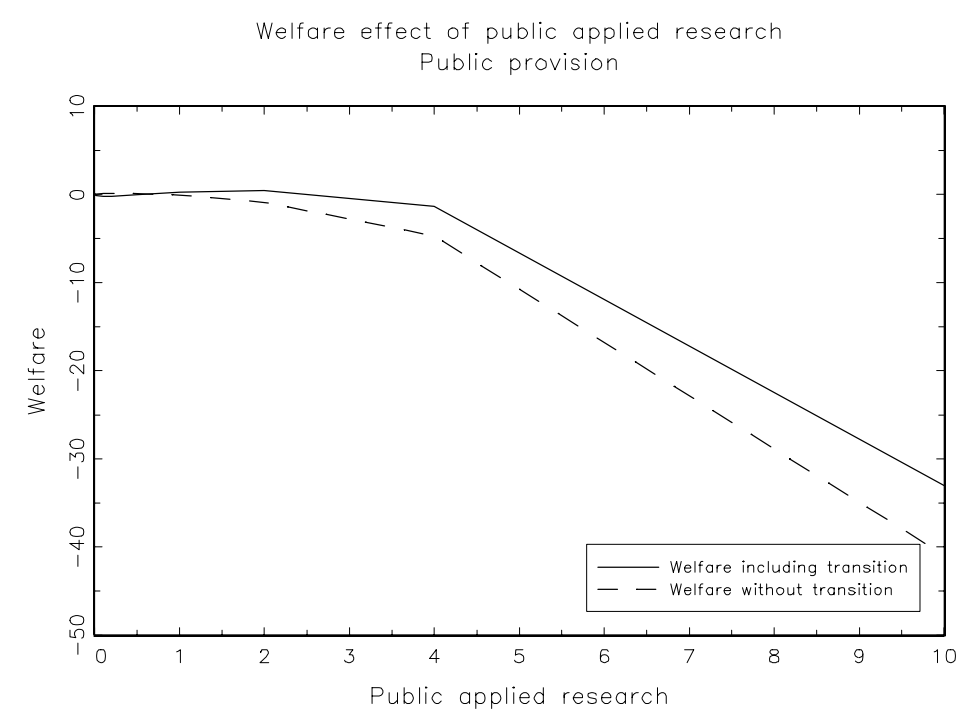

Figure 4: Welfare effect of public applied research 
Table 5.

Welfare effect of basic and applied research when $\Gamma_{b}=\frac{b \Gamma-1}{2 b}$ and $\Gamma_{a}=\frac{b \Gamma+1}{2 b}$.

\begin{tabular}{|c|c|c|c|c|}
\hline$\Gamma$ & $\frac{d c}{d \Gamma}$ & $\frac{d V_{s}}{d \Gamma}$ & $\frac{d V}{d \Gamma}$ & $n$ \\
\hline 0.2 & -0.06 & -0.00 & 0.06 & 6.84 \\
0.5 & -0.07 & -0.00 & 0.06 & 6.55 \\
1 & -0.08 & -0.00 & 0.07 & 6.05 \\
1.5 & -0.09 & 0.00 & 0.09 & 5.57 \\
2 & -0.11 & 0.01 & 0.12 & 5.08 \\
2.5 & -0.13 & 0.01 & 0.15 & 4.60 \\
3 & -0.16 & 0.01 & 0.18 & 4.12 \\
4 & -0.27 & 0.02 & 0.30 & 3.18 \\
5 & -0.47 & 0.02 & 0.50 & 2.28 \\
6 & -0.91 & -0.03 & 0.92 & 1.47 \\
7 & -1.67 & -0.35 & 1.44 & 0.85 \\
8 & -2.30 & -1.42 & 1.29 & 0.48 \\
9 & -2.49 & -3.62 & -0.07 & 0.30 \\
10 & -2.45 & -7.68 & -3.00 & 0.20 \\
\hline
\end{tabular}

Welfare effect of public research

Applied and basic research keeping private optimal proportions

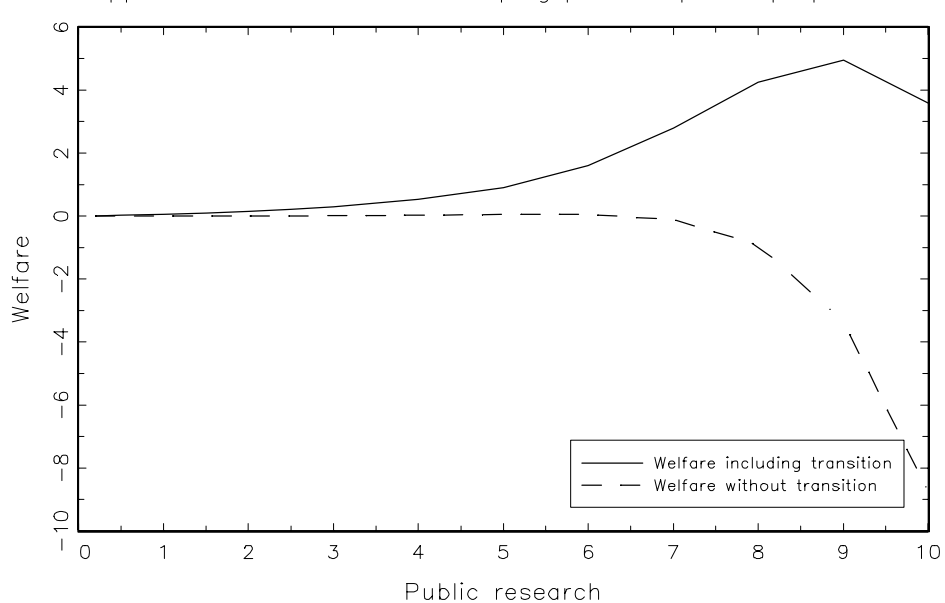

Figure 5: Welfare effect of public research when $\Gamma_{a}=\frac{1+b \Gamma}{2 b}$ and $\Gamma_{b}=\frac{b \Gamma-1}{2 b}$. 


\section{C.2 Public funding of research}

The calibration is made with the same set of parameters as before but using the corresponding equations for this assumption.

Table 6. Welfare effect of the research subsidy

\begin{tabular}{|c|c|c|c|}
\hline$s_{n}$ & $\frac{d c}{d s_{n}}$ & $\frac{d V_{s}}{d s_{n}}$ & $\frac{d V}{d s_{n}}$ \\
\hline 0.0 & -15.35 & -1.53 & 13.77 \\
0.1 & -16.93 & -5.35 & 12.17 \\
0.2 & -18.88 & -12.74 & 7.88 \\
0.3 & -21.34 & -26.85 & -2.76 \\
0.4 & -24.59 & -59.80 & -30.60 \\
0.5 & -29.06 & -156.9 & -120.2 \\
0.6 & -35.72 & -748.2 & -699.8 \\
\hline
\end{tabular}

Welfare effect of the research subsidy

under public funding

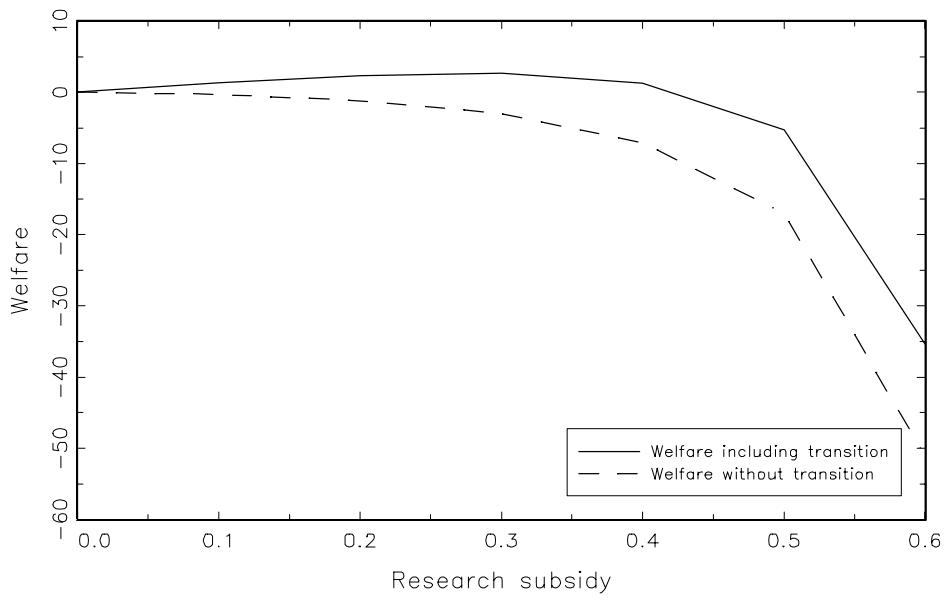

Figure 6: Welfare effect of the research subsidy under public funding. 
Table 7. Welfare effect of public research.

\begin{tabular}{|c|c|c|c|}
\hline$\Gamma$ & $\frac{d c}{d \Gamma}$ & $\frac{d V_{s}}{d \Gamma}$ & $\frac{d V}{d \Gamma}$ \\
\hline 0 & -2.15 & -0.21 & 1.93 \\
0.5 & -2.11 & -0.49 & 1.66 \\
1 & -2.08 & -0.83 & 1.35 \\
1.5 & -2.04 & -1.23 & 0.99 \\
2 & -2.01 & -1.72 & 0.54 \\
2.5 & -1.98 & -2.31 & 0.00 \\
3 & -1.95 & -3.04 & -0.65 \\
4 & -1.89 & -5.11 & -2.51 \\
6 & -1.78 & -15.52 & -11.9 \\
\hline
\end{tabular}

Welfare effect of public funding of research

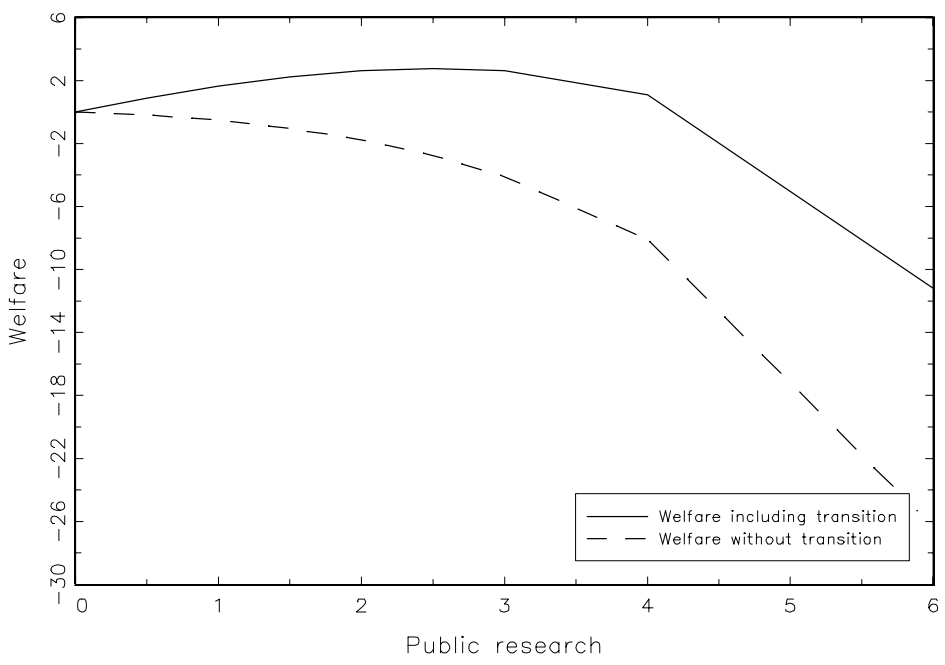

Figure 7: Welfare effect of public R\&D funding. 


\section{Results when private firms do not invest in basic research}

In order to guarantee that the equilibrium value of basic research intensity $n_{b}$ is positive we have to impose some restrictions on the parameters. For the general case without any type of public research, the equations determining $n$ and $k$ in a steady state would be (7) and (8) but substituting the interest rate by its steady state expression

$$
r=\rho+\gamma .
$$

Solve for $k$ in (7) and (8) and note that the first one is increasing in $n$ while the second is decreasing. Therefore, the following condition implies $n>\frac{1}{b}$ :

$$
\chi_{5}<\chi_{6},
$$

where

$$
\begin{aligned}
& \chi_{5}=\left(\frac{\left(1-s_{n}\right)\left(\gamma\left(\frac{1}{b}\right)+\rho+\lambda p\left(\frac{1}{b}\right)\right)}{\lambda b p\left(\frac{1}{b}\right)(1-\alpha) \alpha L^{1-\alpha}}\right)^{\frac{1}{\alpha}} \\
& \chi_{6}=\left(\frac{\alpha^{2} L^{1-\alpha}}{\gamma\left(\frac{1}{b}\right)+\rho+\delta+\tau_{k}}\right)^{\frac{1}{1-\alpha}},
\end{aligned}
$$

and where $\gamma\left(\frac{1}{b}\right)$ is the growth rate (see equation (20) for its functional form) associated to $n=\frac{1}{b}$ and $p(n)=\frac{1+b n}{2 \sqrt{b}}$.

Notice that the introduction of the public research will modify the condition for positive private basic research given in equation (47). If we consider public provision of research, the condition is

$$
\chi_{7}<\chi_{8},
$$

where

$$
\begin{aligned}
& \chi_{7}=\left(\frac{\left(1-s_{n}\right)\left(\gamma\left(\frac{1}{b}\right)+\rho+\lambda p\left(\frac{1}{b}\right)+\lambda\left(\Gamma_{a}\left(1+b \Gamma_{b}\right)\right)^{\frac{1}{2}}\right)}{\lambda b p\left(\frac{1}{b}\right)(1-\alpha) \alpha L^{1-\alpha}}\right)^{\frac{1}{\alpha}} \\
& \chi_{8}=\left(\frac{\alpha^{2} L^{1-\alpha}}{\gamma\left(\frac{1}{b}\right)+\rho+\delta+\tau_{k}}\right)^{\frac{1}{1-\alpha}} .
\end{aligned}
$$

Similarly, if we consider public funding of research projects, the condition for $n_{b}$ positive is obtained from equations (21) and (23) obtained in section 3. Following the same reasoning used to derive condition (47) a sufficient condition for $n_{b}$ to be positive is

$$
\chi_{9}<\chi_{10}
$$

where

$$
\begin{aligned}
\chi_{9} & =\left(\frac{\left(1-s_{n}\right)\left(\gamma\left(\frac{1}{b}+\Gamma_{b}-\Gamma_{a}\right)+\rho+\lambda p\left(\frac{1}{b}, \Gamma\right)\right)}{\lambda b p\left(\frac{1}{b}, \Gamma\right)(1-\alpha) \alpha L^{1-\alpha}}\right)^{\frac{1}{\alpha}} \\
\chi_{10} & =\left(\frac{\alpha^{2} L^{1-\alpha}}{\gamma\left(\frac{1}{b}+\Gamma_{b}-\Gamma_{a}\right)+\rho+\delta+\tau_{k}}\right)^{\frac{1}{1-\alpha}}
\end{aligned}
$$


and where $\gamma\left(\frac{1}{b}+\Gamma_{b}-\Gamma_{a}\right)$ is the rate of growth associated to a level of research intensity $n=\frac{1}{b}+\Gamma_{b}-\Gamma_{a}$. The following subsections show the results when these conditions are not satisfied.

\section{D.1 Public provision of research}

If the equilibrium level of private research is not large enough (i.e. $n \leq \frac{1}{b}$ ), firms do not invest in basic research and therefore, $n_{a}=n$ and $n_{b}=0$. Consequently, the arrival rate of innovations in the private sector will be given by $\lambda n^{\frac{1}{2}}$ and the research arbitrage equation will now be

$$
1-s_{n}=\left(\frac{\lambda}{n_{t}^{\frac{1}{2}}}\right)\left(\frac{(1-\alpha) \alpha L^{1-\alpha} k_{t}^{\alpha}}{r_{t}+\lambda n_{t}^{\frac{1}{2}}+\lambda\left(\Gamma_{a}\left(1+b \Gamma_{b}\right)\right)^{\frac{1}{2}}}\right) .
$$

The equations determining the steady state value of $n$ and $k$ are given by

$$
\begin{aligned}
\gamma+\rho+\delta+\tau_{k} & =\alpha^{2} L^{1-\alpha} k^{\alpha-1} \\
1-s_{n} & =\left(\frac{\lambda}{n_{t}^{\frac{1}{2}}}\right)\left(\frac{\lambda(1-\alpha) \alpha L^{1-\alpha} k_{t}^{\alpha}}{r_{t}+\lambda n_{t}^{\frac{1}{2}}+\lambda\left(\Gamma_{a}\left(1+b \Gamma_{b}\right)\right)^{\frac{1}{2}}}\right) .
\end{aligned}
$$

Following the same steps as in the proofs of propositions 1, 2 and 3 we can compute the derivatives of private research and growth with respect to the policy variables. We find that these derivatives are given by

$$
\begin{aligned}
\frac{d n}{d s_{n}} & =-\frac{\left(\frac{(1-\alpha) \zeta}{k}\right)\left(\gamma+\rho+\lambda n^{\frac{1}{2}}+\lambda \Gamma_{a}\left(1+b \Gamma_{b}\right)\right)}{\operatorname{det}\left(J_{F}\right)} \\
\frac{d n}{d \Gamma_{a}} & =\frac{(1-\alpha) \zeta\left(\frac{\beta \gamma}{n_{A}}\left(\frac{\lambda}{n^{\frac{1}{2}}}+\frac{\left(1-s_{n}\right)}{k}\right)+\left(\frac{\left(1-s_{n}\right) \lambda}{k}\right)\left(\frac{1+b \Gamma_{b}}{\Gamma_{a}}\right)^{\frac{1}{2}}\right)}{\operatorname{det}\left(J_{F}\right)} \\
\frac{d n}{d \Gamma_{b}} & =\frac{(1-\alpha) \zeta\left(\frac{(1-\beta) \gamma}{n_{B}}\left(\frac{\lambda}{n^{\frac{1}{2}}}+\frac{\left(1-s_{n}\right)}{k}\right)+\left(\frac{\left(1-s_{n}\right) \lambda b}{2 k}\right)\left(\frac{\Gamma_{a}}{1+b \Gamma_{b}}\right)^{\frac{1}{2}}\right)}{\operatorname{det}\left(J_{F}\right)}
\end{aligned}
$$

and

$$
\begin{aligned}
\frac{d \gamma}{d s}= & \left(\frac{\beta \gamma}{n_{A}}\right) \frac{d n}{d s} \\
\frac{d \gamma}{d \Gamma_{a}}= & \left(\frac{\beta \gamma}{n_{A}}\right)\left(\frac{(1-\alpha) \zeta}{-\operatorname{det}\left(J_{F}\right)}\right)\left(\frac{\lambda\left(1-s_{n}\right)}{2 k n^{\frac{1}{2}}}+\frac{\lambda \pi}{2 k n^{\frac{3}{2}}}-\left(\frac{\lambda\left(1-s_{n}\right)}{2 k}\right)\left(\frac{1+b \Gamma_{b}}{\Gamma_{a}}\right)^{\frac{1}{2}}\right) \\
\frac{d \gamma}{d \Gamma_{b}}= & \left(\frac{(1-\beta) \gamma}{n_{B}}\right)\left(\frac{(1-\alpha) \zeta}{-\operatorname{det}\left(J_{F}\right)}\right) \\
& \left(\frac{\lambda\left(1-s_{n}\right)}{2 k n^{\frac{1}{2}}}+\frac{\lambda \pi}{2 k n^{\frac{3}{2}}}-\left(\frac{\beta n_{B}}{(1-\beta) n_{A}}\right)\left(\frac{\lambda b\left(1-s_{n}\right)}{2 k}\right)\left(\frac{\Gamma_{a}}{1+b \Gamma_{b}}\right)^{\frac{1}{2}}\right) .
\end{aligned}
$$

Since $\operatorname{det}\left(J_{F}\right)$ is negative, the derivatives of private research with respect to the subsidy rate and basic and applied public research are respectively positive, negative and negative. This implies that the derivative of the growth rate with respect to the research subsidy is positive. The relationship between steady state growth and public research is ambiguous. We find that $\frac{d \gamma}{d \Gamma_{a}}$ will be positive whenever

$$
\Gamma_{a}>\frac{1+b \Gamma_{b}}{b}
$$


since under this condition $\frac{\lambda\left(1-s_{n}\right)}{2 k n^{\frac{1}{2}}}-\left(\frac{\lambda\left(1-s_{n}\right)}{2 k}\right)\left(\frac{1+b \Gamma_{b}}{\Gamma_{a}}\right)^{\frac{1}{2}}$ is positive. A sufficient condition for negativity would be obtained forcing

$$
\frac{\lambda\left(1-s_{n}\right)}{2 k n^{\frac{1}{2}}}+\frac{\lambda \pi}{2 k n^{\frac{3}{2}}}-\left(\frac{\lambda\left(1-s_{n}\right)}{2 k}\right)\left(\frac{1+b \Gamma_{b}}{\Gamma_{a}}\right)^{\frac{1}{2}}<0 .
$$

Of course, a necessary condition is

$$
\Gamma_{a} \leq \frac{1+b \Gamma_{b}}{b}
$$

Requiring (50) is equivalent to require

$$
\left(\frac{\Gamma_{a}}{1+b \Gamma_{b}}\right)^{\frac{1}{2}}<\frac{\lambda n}{2 \lambda n^{\frac{1}{2}}+\rho+\gamma+\lambda\left(\Gamma_{a}\left(1+b \Gamma_{b}\right)\right)^{\frac{1}{2}}} .
$$

Let $\bar{n}$ be the equilibrium value of research intensity when $\Gamma_{a}=0$. Then if the right hand side of (51) is positive when evaluated at 0 , there will exist a range of values of $\Gamma_{a}$ for which the condition is satisfied. However, the left hand side of (51) grows with $\Gamma_{a}$ and the right hand side decreases (because $n$ is negatively related to $\Gamma_{a}$ and the function is increasing in $n$ ). This implies that we will reach a value of $\Gamma_{a}$ smaller than $\frac{1+b \Gamma_{b}}{b}$ for which the condition is no longer satisfied.

With respect to the growth derivative of public basic research, notice that if $\Gamma_{a}=0$, it will be positive for any positive value of public basic research. If $\Gamma_{a}$ is positive, then a sufficient condition for $\frac{d \gamma}{d \Gamma_{b}}>0$ is the following:

$$
\Gamma_{b} \leq \frac{1+\left(1+\left(\frac{\beta}{1-\beta}\right)^{2}\right)^{\frac{1}{2}}}{\frac{b}{2}\left(\frac{\beta}{1-\beta}\right)^{2}} .
$$

In order to obtain $\frac{d \gamma}{d \Gamma_{b}}<0$ the next inequality must be satisfied:

$$
\frac{\lambda\left(1-s_{n}\right)}{2 k n^{\frac{1}{2}}}+\frac{\lambda \pi}{2 k n^{\frac{3}{2}}}-\left(\frac{\beta}{1-\beta}\right) \frac{n_{B}}{n_{A}}\left(\frac{\lambda b\left(1-s_{n}\right)}{2 k}\right)\left(\frac{\Gamma_{a}}{1+b \Gamma_{b}}\right)^{\frac{1}{2}}<0,
$$

which is equivalent to require

$$
b \Gamma_{b}\left(\frac{\beta}{1-\beta}\right)\left(\frac{\Gamma_{a}}{1+b \Gamma_{b}}\right)^{\frac{1}{2}}>\frac{\left(2 \lambda n^{\frac{1}{2}}+\rho+\gamma+\lambda\left(\Gamma_{a}\left(1+b \Gamma_{b}\right)\right)^{\frac{1}{2}}\right)\left(n+\Gamma_{a}\right)}{n} .
$$

Of course, for (53) to be satisfied it is necessary that (52) is not. The right hand side of (53) is decreasing in $n$ if $\Gamma_{a}>n$. Therefore, if we impose $\Gamma_{a}>\frac{1}{b}$ and recall that we are just considering equilibria with $n<\frac{1}{b}$, we can consider this expression decreasing in $n$. Therefore, a sufficient condition for $\frac{d \gamma}{d \Gamma_{b}}<0$ would be

$$
b \Gamma_{b}\left(\frac{\beta}{1-\beta}\right)\left(\frac{\Gamma_{a}}{1+b \Gamma_{b}}\right)^{\frac{1}{2}}>\frac{\left(2 \lambda(\hat{n})^{\frac{1}{2}}+\rho+\gamma(\hat{n})+\lambda\left(\Gamma_{a}\right)^{\frac{1}{2}}\right)\left(\hat{n}+\Gamma_{a}\right)}{\hat{n}},
$$

where $\hat{n}$ is the equilibrium value of research intensity when $\Gamma_{b}=0$.

Therefore, if initially private firms do not perform basic research, the appropriate policy to induce them to do so consists of research subsidies that will increase the level of private research since the effect of public $\mathrm{R} \& \mathrm{D}$, though positive on growth under some conditions, reduces the total amount of private research and thus, will not induce a positive level of private basic research. 


\section{D.2 Public funding}

When private research does not reach a high enough level, private firms may not devote resources to basic research. In the case of the public funding assumption this decision depends also on the level of public research. If $n<\frac{1}{b}+\Gamma_{b}-\Gamma_{a}$, then all the research resources of private firms are devoted to applied projects of research. In this case, the arrival rate of innovations will be

$$
\lambda\left(\left(n+\Gamma_{a}\right)\left(1+b \Gamma_{b}\right)\right)^{\frac{1}{2}},
$$

while the research arbitrage equation will be given by

$$
1-s_{n}=\left(\frac{\lambda\left(\left(n_{t}+\Gamma_{a}\right)\left(1+b \Gamma_{b}\right)\right)^{\frac{1}{2}}}{n_{t}}\right)\left(\frac{(1-\alpha) \alpha L^{1-\alpha} k_{t}^{\alpha}}{r_{t}+\lambda\left(\left(n_{t}+\Gamma_{a}\right)\left(1+b \Gamma_{b}\right)\right)^{\frac{1}{2}}}\right) .
$$

Therefore, the derivatives of private research and the rate of growth may be computed to obtain

$$
\begin{aligned}
\frac{d n}{d s} & =-\frac{\left(\frac{(1-\alpha) \zeta}{k}\right)\left(\gamma+\rho+\lambda\left(\left(\Gamma_{a}+n\right)\left(1+b \Gamma_{b}\right)\right)^{\frac{1}{2}}\right)}{\operatorname{det}\left(J_{F}\right)} \\
\frac{d n}{d \Gamma_{a}} & =\frac{(1-\alpha) \zeta\left(\left(\frac{\beta \gamma}{n_{A}}\right)\left(\frac{\lambda\left(\left(n+\Gamma_{a}\right)\left(1+b \Gamma_{b}\right)\right)^{\frac{1}{2}}}{n}+\frac{\left(1-s_{n}\right)}{k}\right)-\frac{\left(1-s_{n}\right)(\rho+\gamma)}{2 k\left(n+\Gamma_{a}\right)}\right)}{\operatorname{det}\left(J_{F}\right)} \\
\frac{d n}{d \Gamma_{b}} & =\frac{(1-\alpha) \zeta\left(\left(\frac{(1-\beta) \gamma}{n_{B}}\right)\left(\frac{\lambda\left(\left(n+\Gamma_{a}\right)\left(1+b \Gamma_{b}\right)\right)^{\frac{1}{2}}}{n}+\frac{\left(1-s_{n}\right)}{k}\right)+\left(\frac{\left(1-s_{n}\right) \lambda b}{2 k}\right)\left(\frac{\Gamma_{a}+n}{1+b \Gamma_{b}}\right)^{\frac{1}{2}}-\left(\frac{b \lambda \pi}{2 n k}\right)\left(\frac{\Gamma_{a}+n}{1+b \Gamma_{b}}\right)^{\frac{1}{2}}\right)}{\operatorname{det}\left(J_{F}\right)},
\end{aligned}
$$

and

$$
\begin{aligned}
\frac{d \gamma}{d s_{n}}= & \left(\frac{\beta \gamma}{n_{A}}\right) \frac{d n}{d s} \\
\frac{d \gamma}{d \Gamma_{a}}= & \left(\frac{\beta \gamma}{n_{A}}\right)\left(\frac{(1-\alpha) \zeta}{-\operatorname{det}\left(J_{F}\right)}\right)\left(\frac{\lambda\left(1-s_{n}\right)}{k}\left(\frac{d p(n)}{d n}\right)-\lambda \frac{d}{d n}\left(\frac{p(n)}{n}\right) \frac{\tilde{\pi}}{k}+\frac{\left(1-s_{n}\right)(\rho+\gamma)}{k n_{A}}\right) \\
\frac{d \gamma}{d \Gamma_{b}}= & \left(\frac{(1-\beta) \gamma}{n_{B}}\right)\left(\frac{(1-\alpha) \zeta}{-\operatorname{det}\left(J_{F}\right)}\right) \\
& \left(\left(\frac{\lambda\left(1-s_{n}\right)}{k}\right)\left(\frac{d p(n)}{d n}\right)-\lambda \frac{d}{d n}\left(\frac{p(n)}{n}\right) \frac{\tilde{\pi}}{k}+\left(\frac{\beta}{1-\beta}\right)\left(\frac{\left(1-s_{n}\right) b \Gamma_{b}(\rho+\gamma)}{2 k n_{A}\left(1+b \Gamma_{b}\right)}\right)\right),
\end{aligned}
$$

where

$$
\begin{aligned}
p(n) & =\left(\left(n+\Gamma_{a}\right)\left(1+b \Gamma_{b}\right)\right)^{\frac{1}{2}} \\
\frac{d p(n)}{d n} & =\frac{1}{2}\left(\frac{1+b \Gamma_{b}}{n+\Gamma_{a}}\right)^{\frac{1}{2}} \\
\frac{d}{d n}\left(\frac{p(n)}{n}\right) & =-\frac{1}{2}\left(\frac{\left(n+2 \Gamma_{a}\right)\left(1+b \Gamma_{b}\right)^{\frac{1}{2}}}{n^{2}\left(n+\Gamma_{a}\right)^{\frac{1}{2}}}\right) .
\end{aligned}
$$

Therefore, the three derivatives are positive as we obtained for the case in which private firms performed basic research. 